\title{
Long-Term Field Evaluation Reveals Huanglongbing Resistance in Citrus Relatives
}

\author{
Chandrika Ramadugu, University of California Riverside, Riverside, CA; Manjunath L. Keremane, USDA-ARS, National Clonal Germ- \\ plasm Repository for Citrus and Dates, Riverside, CA; Susan E. Halbert, Division of Plant Industry, Gainesville, FL; Yong Ping Duan, U.S. \\ Horticultural Research Laboratory, Fort Pierce, FL; Mikeal L. Roose, University of California Riverside; Ed Stover, U.S. Horticultural \\ Research Laboratory; and Richard F. Lee, USDA-ARS, National Clonal Germplasm Repository for Citrus and Dates
}

\begin{abstract}
Ramadugu, C., Keremane, M. L., Halbert, S. E., Duan, Y. P., Roose, M. L., Stover, E., and Lee, R. F. 2016. Long-term field evaluation reveals huanglongbing resistance in Citrus relatives. Plant Dis. 100:1858-1869.

Citrus huanglongbing (HLB) is a destructive disease with no known cure. To identify sources of HLB resistance in the subfamily Aurantioideae to which citrus belongs, we conducted a six-year field trial under natural disease challenge conditions in an HLB endemic region. The study included 65 Citrus accessions and 33 accessions belonging to 20 other closely related genera. For each accession, eight seedling trees were evaluated. Based on quantitative polymerase chain reaction analysis of the pathogen titers and disease symptoms, eight disease-response categories were identified. We report two immune, six resistant, and 14 tolerant accessions. Resistance and tolerance observed in different accessions may

be attributed to a multitude of factors, including psyllid colonization ability, absence of pathogen multiplication, transient replication of the bacterium, lack of pathogen establishment in the plant, delayed infection, or recovery from infection. Most citrus cultivars were considered susceptible: 15 citrons, lemons, and limes retained leaves in spite of the disease status. Resistance and high levels of field tolerance were observed in many noncitrus genera. Disease resistance/tolerance was observed in Australian citrus relative genera Eremocitrus and Microcitrus, which are sexually compatible with citrus and may be useful in future breeding trials to impart HLB resistance to cultivated citrus.
\end{abstract}

Citrus huanglongbing (HLB) or greening is a formidable disease that has caused enormous economic loss to many citrus growing regions of the world (Bové 2006; da Graça and Korsten, 2004; Halbert and Manjunath 2004). HLB has been in India and China for over a hundred years (Husain and Nath 1927; Chen 1943). The disease was first reported in the Western hemisphere about 12 years ago (ColettaFilho et al. 2004; Halbert 2005). HLB is associated with an unculturable $\alpha$-proteobacterium, 'Candidatus Liberibacter' and is transmitted by two psyllid vectors, Diaphorina citri Kuwayama and Trioza erytreae (del Guercio). At least three species of the bacterium, ' $C a$. L. asiaticus', ' $\mathrm{Ca}$. L. africanus', and ' $\mathrm{Ca}$. L. americanus', are known to be associated with HLB. Of the three species, ' $\mathrm{Ca}$. L. asiaticus' is the most virulent and widely distributed worldwide.

In the 10 years since its introduction, the Florida citrus industry has lost over $50 \%$ of its citrus and production is decreasing at an alarming rate (Gottwald 2010; USDA National Agricultural Statistics Service 2015). In California, the vector Diaphorina citri (Asian citrus psyllid [ACP]) was first reported near the border with Mexico, near San Diego in 2008. Based on the Florida experience as well as recently developed research information, California was proactive and established several regulatory and operational guidelines to prevent, eradicate, or delay the introduction and spread of both ACP and HLB. Consequently, the rate of vector and disease spread has been significantly reduced in California compared with Florida. However, neither the preventive efforts nor the management practices currently being followed in HLB endemic regions of the world are sufficient for the long-term survival of an economically feasible and environmentally affordable citrus industry. Development of resistance to

Corresponding author: C. Ramadugu; E-mail: chandram@ucr.edu

C. Ramadugu and M. L. Keremane contributed equally to this work.

*The $\boldsymbol{e}$-Xtra logo stands for "electronic extra" and indicates that one supplementary table is published online.

Accepted for publication 12 April 2016.

http://dx.doi.org/10.1094/PDIS-03-16-0271-RE

(C) 2016 The American Phytopathological Society
HLB, either through traditional breeding, by transgenic approaches, or both appears to be the best long-term solution for survival of the citrus industry.

HLB-resistant citrus cultivars are not reported in the literature. Most of the early work on cultivar assessment was based on symptomatology. Interpretation of the disease evaluation data were often difficult because of similarities to other disease symptoms and poor cultural practices resulting in nutritional deficiencies that could be mistaken for HLB. In addition, a reliable laboratory diagnostic method for the agent associated with HLB was not available before 2005 Many rutaceous plants carrying HLB-associated bacteria may not develop characteristic HLB symptoms, such as blotchy leaf mottle, loss of foliage, and lopsided fruit. Disease evaluation studies conducted in recent times utilize nucleic acid-based detection of the HLBassociated bacterium. Detection of the pathogen at a single time point may not be adequate to understand the HLB disease status of the taxa. Many citrus relatives appear to support the replication of Liberibacters for a short period of time before suppressing them completely. Indian wood apple (Limonia acidissima; syn. Feronia acidissima) is reported to be a transient host of ' $\mathrm{Ca}$. L. asiaticus' (Hung et al. 2000; Koizumi et al. 1996). Murraya species are reported to have very low titers of 'Ca. L. asiaticus' and are reported as possible transient hosts of Liberibacter spp. (Beattie et al. 2012; Walter et al. 2012a).

Inconsistency in published reports regarding disease response of citrus cultivars to HLB can be confusing; the variability observed may be due to host genotypes, geographical location, cultural practices, or differences in pathogenicity of diverse liberibacter isolates. Pummelos from many Asian countries were reported to be HLB resistant, but recent studies from the same geographic regions report that pummelos are susceptible (Ahmad et al. 2008; Shokrollah et al. 2009). HLB-resistant pummelos are still present in Vietnam and India (M. L. Keremane, personal observation). Murraya paniculata has been reported as HLB resistant from Taiwan (Hung et al. 2000,2001 ) but as susceptible to both ' $\mathrm{Ca}$. L. asiaticus' and ' $\mathrm{Ca}$. L. americanus' from Brazil (Lopes et al. 2010).

Citrus has been cultivated for many centuries. Both clonal propagation and nucellar embryony (a form of apomixis) have resulted in very low genetic diversity within cultivated citrus types. Wild relatives of plants that are genetically related to many cultivated crops often possess resistance to plant diseases. In stone fruits, hybrid root 
stocks developed using wild relatives of prune are currently used extensively for cultivation of prune, peach, plum, apricot, and almond (Guajardo et al. 2015). Resistance to quick decline caused by the citrus tristeza virus prompted the widespread use of both trifoliate orange, and its hybrids with citrus as rootstocks. Incorporating useful traits from exotic germplasm can provide solutions to crucial disease problems and alleviate genetic vulnerability (Ford-Lloyd et al. 2011; Holland 2004).

HLB-tolerant cultivars have been identified by various research groups around the world (Koizumi et al. 1993; Shokrollah et al. 2009; Stover and McCollum 2011; Stover et al. 2014). Identification and analysis of HLB resistance in citrus relatives can be valuable both for understanding the mechanisms of resistance as well as for breeding trials. We have conducted an HLB evaluation trial using material from the Citrus Variety Collection (CVC), University of California, Riverside, CA, which has over 1,200 cataloged accessions belonging to the subfamily Aurantioideae. Research and propagation material for many of these accessions are available to researchers throughout the world from the United States Department of Agriculture (USDA), Agricultural Research Service, National Clonal Germplasm Repository for Citrus and Dates, Riverside, CA. Details of each genotype are available from the website (http://www. citrusvariety.ucr.edu).

In this study, a long-term field evaluation was conducted to understand the responses of various cataloged genotypes of citrus and citrus relatives to prevalent liberibacter populations associated with HLB in Florida under natural challenge conditions. Any valuable resistance identified in this trial can be traced back to individual trees for further research. The use of seedlings avoided possible influence of rootstocks on disease response; open pollinated seeds increased the probability of introducing variability within each accession, especially in accessions where apomixis does not exist.

The definition of resistance varies in different pathosystems. In general, a resistant plant will have a much lower level of the pathogen compared with a susceptible plant and will not be as symptomatic. Susceptible taxa typically have a higher pathogen titer and will display symptoms. Tolerance is difficult to measure; tolerant plants may have typical disease symptoms but continue to produce fruit. Immunity is defined as a state where the pathogen is never detected in the plant even though it is exposed to the pathogen (Vale et al. 2001).

The main objective of the current study was to identify sources of HLB tolerance or resistance, or both, in citrus cultivars and close relatives belonging to the subfamily Aurantioideae. Since HLB is known to have a long incubation period, the plant hosts often have low pathogen titers and the bacterium is irregularly distributed within the plant host. Hence, we conducted the field trial for a period of six years to enable accurate evaluation of the test accessions. We had three objectives: i) to identify patterns of disease response in cataloged accessions of the plant hosts; ii) to identify sources of disease resistance and tolerance under field conditions in citrus and closely related genera; and iii) to compare HLB incidence over time in specific accessions, with psyllid infestation data that was generated in a different study using the same set of plants. To our knowledge, this is a first report of a large, replicated trial representing genetically diverse accessions that have been systematically screened for the purpose of identifying immune, resistant, and HLB tolerant germplasm.

\section{Materials and Methods}

Source of plant materials, experimental design. The majority of the taxa used for the field trial were cataloged accessions obtained from the CVC (http://www.citrusvariety.ucr.edu). Seeds were collected from 108 accessions of citrus and citrus relatives belonging to the subfamily Aurantioideae, family Rutaceae. Plants were selected from the core collection to represent about $85 \%$ of the genetic diversity present in the entire collection (Barkley 2003). Seeds for Afraegle paniculata (Schum.) Engl., Aegle marmelos (L.) Corr., and Casimiroa edulis La Llave \& Lex were obtained from the Fruit and Spice Park, Miami, FL. Seeds of Zanthoxylum ailanthoides were obtained from the University of Georgia. Plant maintenance in the greenhouses was as described in a previous publication (Westbrook et al. 2011). Briefly, the seeds were germinated in cone-tainers in greenhouses located in the U.S. Horticultural Research Laboratory, Fort Pierce, FL. Six- to nine-month-old seedlings were planted in the field in a randomized complete block design in July 2009. Trees were planted with $0.6-\mathrm{m}$ spacing. The field plants were irrigated and fertilized but pesticides were not applied during the study, to encourage psyllid survival, feeding, colonization, and subsequent infection of the plants. Maintenance of the field trees has been described elsewhere (Richardson et al. 2011). The HLB disease pressure was considered to be very high in Pico's farm, Fort Pierce, FL, where the field trial was conducted. The psyllid populations were adequate for conducting a HLB evaluation trial under natural disease spread conditions (Richardson et al. 2011, Westbrook et al. 2011).

Sample collection and DNA extraction. Twigs from three or more branches were collected from individual trees for further processing. If disease symptoms were observed, samples were collected from branches suspected to have the pathogen. We used both petioles (from five to 10 leaves) and bark pieces for the DNA extractions. About $100 \mathrm{mg}$ of chopped tissue was placed in 2-ml screwcap tubes with O-rings (Thermo Fisher Scientific, Fair Lawn, NJ). About 8 to 10 zirconium beads (Glen Mills, Inc., Clifton, NJ) of $2.5 \mathrm{~mm}$ diameter were added, the samples were lyophilized overnight, and were then homogenized with extraction buffer (Qiagen, Inc., Valencia, CA) in a bead beater (Biospec Products Inc., Bartlesville, OK). At the beginning of the field trial, samples were processed at the U.S. Horticultural Research laboratory, Fort Pierce, FL, using Plant DNeasy kit (Qiagen, Inc.) according to the manufacturer's instructions. The DNA extractions were shipped to the USDA Riverside laboratory for further analysis. In the last two years of the experiment (2014 and 2015), about $500 \mathrm{mg}$ of petiole, midrib, and shoot samples were placed in 2-ml screwcap tubes, filled with $95 \%$ alcohol, and were shipped to Riverside under a valid USDA APHIS (Animal and Plant Health Inspection Service) moving permit. Alcoholpreserved samples were carefully removed from the tubes, airdried briefly for about $3 \mathrm{~min}$, and, using a razor blade, about $100 \mathrm{mg}$ of sample representing petiole, midrib, and bark tissue was chopped and added to 96 individual wells of a deep-well plate (USA Scientific Inc., Ocala, FL) along with $2.5 \mathrm{~mm}$ of zirconium beads (about 8 to 10) and covered with clustercaps (USA Scientific Inc., Ocala, FL). In each 96-well plate, four wells (F12 to H12) were designated for positive and negative controls in polymerase chain reaction (PCR). In addition, three to five randomly selected wells without plant samples served as negative extraction controls. Care was taken to avoid cross contaminations by changing gloves between samples. Plant material was removed using forceps and all tools were bleached for a minimum of $3 \mathrm{~min}$ between samples, followed by a sterile water rinse. The lyophilized samples were powdered using a bead beater twice, $3 \mathrm{~min}$ each, and centrifuged for $5 \mathrm{~min}$ at $3,750 \times g$. Cluster caps from individual columns were removed carefully and $500 \mu$ l of RLT buffer (Qiagen, Inc.) was added. The caps were replaced and the samples were ground again, using the bead beater as described above. Further processing was done robotically, according to the manufacturer's instructions, in a KingFisher flex magnetic particle processor (Thermo Scientific), and the extracted DNA was eluted in a final volume of $100 \mu \mathrm{l}$ of AE buffer (Qiagen, Inc.).

Quantitative (q)PCR methods. We used a TaqMan-based multiplex real-time qPCR method for detection of the 16S rDNA gene from ' $\mathrm{Ca}$. L. asiaticus' and the mitochondrial gene cytochrome oxidase (COX) from the plant hosts ( $\mathrm{Li}$ et al. 2006; Manjunath et al. 2008; Razi et al. 2014). Cycle threshold (Ct) values for the COX gene were useful to verify quality of DNA extractions. Inclusion of positive plasmid control samples and negative extraction controls was useful in maintaining rigorous quality control during extractions and qPCR assays. A Stratagene Mx3005P qPCR machine (Agilent Technologies, Santa Clara, CA) and an Applied Biosystems ViiA 7 real-time PCR system (Thermo Scientific) were utilized for the qPCR assays. 
To confirm tolerance/resistance recorded in several individual trees, five to 10 additional samples were collected from multiple branches, DNA extractions and qPCR were conducted separately for the different samples. Only one value for each time point (the lowest $\mathrm{Ct}$ recorded by qPCR analysis) is indicated in the data tables (Supplementary Table S1).

Plant response categories. Instead of determining if the plants are susceptible or resistant, we assigned plants to eight different categories depending on the qPCR results, intensity of HLB symptoms, and plant growth patterns in the field, as described below.

\section{Results}

HLB disease categories. Most studies describing HLB disease status of citrus and related accessions categorize the host plants as susceptible, tolerant, or resistant. In the current study, which includes heterologous taxa belonging to 21 genera, we identified eight types

Table 1. Taxa evaluated in the field trial and the huanglongbing disease rating based on disease symptoms and pathogen titer, as estimated by real time polymerase chain reaction assays

\begin{tabular}{|c|c|c|c|c|}
\hline No. & Botanical name (CRC number) & Common/cultivar name & Taxonomical group & Category \\
\hline 1 & Clausena excavata Burm. f. (3166) & Pink wampee & Aura/Clauseneae/Clauseninae & 1 \\
\hline 2 & Glycosmis pentaphylla (Retz.) DC. (3285) & Orange berry/Gin berry & Aur/Clauseneae/Clauseninae & 1 \\
\hline 3 & Bergera koenigii L. (3165) & Curry leaf & Aur/Clauseneae/Clauseninae & 2 \\
\hline 4 & Casimiroa edulis La Llave \& Lex & White sapote & Toddalioideae & 2 \\
\hline 5 & $\begin{array}{l}\text { Eremocitrus glauca hybrid (Lindl.) Swingle } \\
\text { (4105) }\end{array}$ & Australian desert lime hybrid & Aur/Citreae/Citrinae & 2 \\
\hline 6 & Murraya paniculata (L.) Jack. (1637) & 'Orange jasmine' & Aur/Clauseneae/Clauseninae & 2 \\
\hline 7 & Naringi crenulata (Roxb.) Nicolson (2879) & Hesperethusa & Aur/Citreae/Citrinae & $2 * b$ \\
\hline 8 & Zanthoxylum ailanthoides Siebold \& Zucc. & Japanese prickly ash & Toddalioideae & 2 \\
\hline 9 & Citrus halimii B.C. Stone (3780) & 'Mountain' citron & Aur/Citreae/Citrinae & 3 \\
\hline 10 & $\begin{array}{l}\text { Microcitrus australasica (F. Muell.) Swing. } \\
\text { (1484) }\end{array}$ & Australian finger lime var. 'Sanguinea' & Aur/Citreae/Citrinae & 3 \\
\hline 11 & $\begin{array}{l}\text { Microcitrus hybrid (M. australis } \times \\
\text { M. australasica) }(1485)\end{array}$ & 'Sydney hybrid' & Aur/Citreae/Citrinae & 3 \\
\hline 12 & $\begin{array}{l}\text { Microcitrus inodora (F.M.Bail) Swing. } \\
\text { (3785) }\end{array}$ & Large-leaf Australian wild lime & Aur/Citreae/Citrinae & $3 *$ \\
\hline 13 & $\begin{array}{l}\text { XMicrocitronella sp. (Microcitrus } \\
\text { australasica } \times \text { Calamondin) }(1466)\end{array}$ & 'Faustrimedin' & Aur/Citreae/Citrinae & 3 \\
\hline 14 & Poncirus trifoliata L. (3549) & 'Simmon's trifoliate' hybrid & Aur/Citreae/Citrinae & 3 \\
\hline 15 & Aegle marmelos (L.) Corr. & Indian bael fruit & Aur/Citreae/Balsamocitrinae & 4 \\
\hline 16 & Aeglopsis chevalieri Swingle (2878) & Chevalier's Aeglopsis & Aur/Citreae/Balsamocitrinae & 4 \\
\hline 17 & Balsamocitrus daweii Stapf. (3514) & Uganda powder flask & Aur/Citreae/Balsamocitrinae & 4 \\
\hline 18 & Citrus latipes (Swing.) Tan. (3052) & 'Khasi' papeda & Aur/Citreae/Citrinae & 4 \\
\hline 19 & $\begin{array}{l}\text { Microcitrus australis (A. Cunn. ex Mudie) } \\
\text { Swingle (3673) }\end{array}$ & Australian round lime & Aur/Citreae/Citrinae & 4 \\
\hline 20 & Murraya paniculata (L.) Jack. (3171) & 'Hawaiian mock orange' var. 'Ovatifoliolata' & Aur/Clauseneae/Clauseninae & 4 \\
\hline 21 & Poncirus trifoliata L. (4007) & 'Little-leaf' trifoliate & Aur/Citreae/Citrinae & 5 \\
\hline 22 & $\begin{array}{l}\text { XCitroncirus sp. (Cleopatra mandarin } \times \\
\text { P. trifoliata) }(3957)\end{array}$ & 'X639' trifoliate hybrid & Aur/Citreae/Citrinae & 5 \\
\hline 23 & Citrus amblycarpa Och. (2485) & 'Nasnaran' mandarin & Aur/Citreae/Citrinae & 6 \\
\hline 24 & Citrus aurantifolia (Christm.) Swing. (2450) & 'India' lime & Aur/Citreae/Citrinae & 6 \\
\hline 25 & Citrus aurantium L. (2717) & 'Olivelands' sour orange & Aur/Citreae/Citrinae & 6 \\
\hline 26 & Citrus aurantium L. (628) & 'Standard' sour orange & Aur/Citreae/Citrinae & 6 \\
\hline 27 & Citrus davaoensis (Wester) Tan. (2427) & 'Davao' lemon & Aur/Citreae/Citrinae & 6 \\
\hline 28 & Citrus excelsa Wester (2317) & 'Limon Real' papeda & Aur/Citreae/Citrinae & 6 \\
\hline 29 & Citrus hassaku hort. ex Tan. (3907) & 'Hassaku' pummelo hybrid & Aur/Citreae/Citrinae & 6 \\
\hline 30 & Citrus hassaku hort ex Tan (3942) & 'Hassaku' pummelo hybrid & Aur/Citreae/Citrinae & 6 \\
\hline 31 & $\begin{array}{l}\text { Citrus hybrid (53-1-16 'Clem' } \times \text { 'Hamlin') } \times \\
\text { Chinotto F1 (3715) }\end{array}$ & Ex-India sour orange hybrid & Aur/Citreae/Citrinae & 6 \\
\hline 32 & Citrus jambhiri Lush. (400) & 'Florida' rough lemon & Aur/Citreae/Citrinae & 6 \\
\hline 33 & Citrus leiocarpa hort. Ex Tan. (3147) & ‘Koji’ mandarin & Aur/Citreae/Citrinae & 6 \\
\hline 34 & Citrus limettioides Tan. (1482) & 'Palestine' sweet lime & Aur/Citreae/Citrinae & 6 \\
\hline 35 & Citrus limon (L.) Burm. f. (3005) & 'Frost Nucellar Eureka' lemon & Aur/Citreae/Citrinae & 6 \\
\hline 36 & Citrus limon (L.) Burm. f. (3593) & 'Interdonato’ lemon & Aur/Citreae/Citrinae & 6 \\
\hline 37 & Citrus limon (L.) Burm. f. (3885) & Iranian lemon & Aur/Citreae/Citrinae & 6 \\
\hline 38 & Citrus limonia (L.) Osbeck (3919) & 'Lamas' lemon & Aur/Citreae/Citrinae & 6 \\
\hline 39 & Citrus limonia Osbeck. (712) & 'Santa Barbara' red lime & Aur/Citreae/Citrinae & 6 \\
\hline 40 & Citrus longispina Wester (2320) & ‘Talamisan'; 'Winged' lime & Aur/Citreae/Citrinae & 6 \\
\hline 41 & Citrus macrophylla Wester (3842) & 'Alemow' hybrid & Aur/Citreae/Citrinae & 6 \\
\hline 42 & Citrus maxima (Burm.) Merr. (2242) & 'Kao Pan’ pummelo & Aur/Citreae/Citrinae & 6 \\
\hline 43 & Citrus maxima (Burm.) Merr. (2248) & 'Kao Panne' pummelo & Aur/Citreae/Citrinae & 6 \\
\hline 44 & Citrus maxima (Burm.) Merr. (3805) & 'Reinking' pummelo & Aur/Citreae/Citrinae & 6 \\
\hline 45 & Citrus medica L. (3523) & 'Diamante' citron & Aur/Citreae/Citrinae & 6 \\
\hline 46 & Citrus medica L. (3546) & 'South Coast Field Station' citron & Aur/Citreae/Citrinae & 6 \\
\hline
\end{tabular}

\footnotetext{
a Aur $=$ Aurantioideae.

b Asterisks $(*)$ indicate insufficient data.
} 
of responses to the HLB challenge. Real time Ct cutoff values were not set to determine the disease categories. Instead, the complete absence of the pathogen (immunity), absence of pathogen colonization in spite of transient replication (resistance), and successful colonization of the pathogen leading to disease symptom expression (susceptibility) were considered in determining disease categories. Tolerant categories included those accessions in which plants were able to recover from disease and in which the onset of disease was delayed. Another category of tolerant accessions exhibited variability among test plants, with some being susceptible and others resistant. Most accessions in the last category were seedlings of known hybrids.

The field trial was first started with 108 accessions and about 886 seedlings. At the end of six years, 419 plants survived. We were able to grow 98 accessions with replications and retrieved meaningful data from about 90 accessions (data obtained from more than one plant in year two). Many noncitrus accessions did not perform well in the field trial, since growth conditions were not optimal and

Table 1. (continued from preceding page)

\begin{tabular}{|c|c|c|c|c|}
\hline No. & Botanical name (CRC number) & Common/cultivar name & Taxonomical group & Category \\
\hline 47 & Citrus medica L. (661) & 'Indian' citron hybrid & Aur/Citreae/Citrinae & 6 \\
\hline 48 & $\begin{array}{l}\text { Citrus neo-aurantium (C.obovoidea }+C . \\
\text { unshiu graft chimera) (3816) }\end{array}$ & 'Kinkoji Unshiu' graft chimera & Aur/Citreae/Citrinae & 6 \\
\hline 49 & Citrus neo-aurantium Tanaka (3611) & 'Konejime' sour orange hybrid & Aur/Citreae/Citrinae & 6 \\
\hline 50 & Citrus reticulata Blanco (3558) & 'Fremont' mandarin & Aur/Citreae/Citrinae & 6 \\
\hline 51 & Citrus sp. (3172) & Unnamed lemon hybrid & Aur/Citreae/Citrinae & 6 \\
\hline 52 & Citrus taiwanica Tan. and Shimada (2588) & 'Nasho Daidai' sour orange & Aur/Citreae/Citrinae & 6 \\
\hline 53 & Citrus volkameriana Osbeck (3050) & 'Volkamer' lemon hybrid & Aur/Citreae/Citrinae & 6 \\
\hline 54 & Citrus webberi Wester (1455) & ‘Kalpi’ papeda & Aur/Citreae/Citrinae & 6 \\
\hline 55 & Severinia buxifolia (Poiret) Tan. (1497) & Chinese box orange & Aur/Citreae/Citrinae & 6 \\
\hline 56 & Severinia buxifolia (Poiret) Tan. (4107) & Chinese box orange & Aur/Citreae/Citrinae & 6 \\
\hline 57 & Severinia buxifolia (Poiret) Ten. (1491) & Chinese box orange & Aur/Citreae/Citrinae & 6 \\
\hline 58 & XCitroncirus sp. (301) & 'Rusk' citrange trifoliate hybrid & Aur/Citreae/Citrinae & 6 \\
\hline 59 & XCitroncirus sp. (3552) & 'S-281' citrangelo & Aur/Citreae/Citrinae & 6 \\
\hline 60 & $\begin{array}{l}\text { XCitroncirus sp. }(C \text {. paradisi 'Duncan' } \times P \text {. } \\
\text { trifoliata })(3771)\end{array}$ & 'Swingle' citrumelo & Aur/Citreae/Citrinae & 6 \\
\hline 61 & Citrus aurantifolia (Christm.) Swing. (3822) & 'Mexican' lime & Aur/Citreae/Citrinae & 7 \\
\hline 62 & Citrus aurantium L. (3929) & 'Goutoucheng' sour orange & Aur/Citreae/Citrinae & 7 \\
\hline 63 & Citrus aurantium L. (3930) & 'Zhuluan' sour orange hybrid & Aur/Citreae/Citrinae & 7 \\
\hline 64 & Citrus benikoji hort. Ex Tan. (3149) & Unnamed tangor & Aur/Citreae/Citrinae & 7 \\
\hline 65 & Citrus intermedia hort. Ex Tan. (3474) & 'Yama-mikan' sour orange & Aur/Citreae/Citrinae & 7 \\
\hline 66 & Citrus limon (L.) Burm. f. (3176) & 'Frost Nucellar Lisbon' lemon & Aur/Citreae/Citrinae & 7 \\
\hline 67 & Citrus limon (L.) Burm. f. (3892) & 'Mesero' lemon & Aur/Citreae/Citrinae & 7 \\
\hline 68 & Citrus lycopersiciformis hort. ex Tan. (3564) & Kokni orange & Aur/Citreae/Citrinae & 7 \\
\hline 69 & Citrus maderasapatna $(3225)$ & 'Kitchli' sour orange hybrid & Aur/Citreae/Citrinae & $7^{\mathrm{b}}$ \\
\hline 70 & Citrus maxima (Burm.) Merr. (3945) & 'Mato Buntan' pummelo & Aur/Citreae/Citrinae & 7 \\
\hline 71 & Citrus maxima (Burm.) Merr. (3959) & 'Egami Buntan' pummelo & Aur/Citreae/Citrinae & 7 \\
\hline 72 & Citrus maxima (Burm.) Merr. (4026) & 'Pomelit' pummelo hybrid & Aur/Citreae/Citrinae & 7 \\
\hline 73 & Citrus paradisi Macf. (3781) & 'Tahitian' pummelo $\times$ 'Star Ruby' grapefruit & Aur/Citreae/Citrinae & 7 \\
\hline 74 & Citrus reticulata Blanco (3018) & 'Dweet' tangor & Aur/Citreae/Citrinae & 7 \\
\hline 75 & Citrus reticulata Blanco (2590) & 'Tien Chieh' mandarin & Aur/Citreae/Citrinae & 7 \\
\hline 76 & Citrus reticulata Blanco (3022) & 'Frua' mandarin & Aur/Citreae/Citrinae & 7 \\
\hline 77 & Citrus reticulata Blanco (3260) & 'Soh Niamtra' mandarin & Aur/Citreae/Citrinae & 7 \\
\hline 78 & Citrus reticulata Blanco (3326) & 'Scarlett Emperor' mandarin & Aur/Citreae/Citrinae & 7 \\
\hline 79 & Citrus reticulata Blanco (3363) & 'Belady' mandarin & Aur/Citreae/Citrinae & 7 \\
\hline 80 & Citrus reticulata Blanco (3752) & 'Som Keowan' mandarin & Aur/Citreae/Citrinae & 7 \\
\hline 81 & Citrus reticulata Blanco (3958) & 'Koster' mandarin & Aur/Citreae/Citrinae & 7 \\
\hline 82 & Citrus reticulata Blanco (4003) & 'Sun Chu Sha' mandarin & Aur/Citreae/Citrinae & 7 \\
\hline 83 & $\begin{array}{l}\text { Citrus reticulata hybrid (Clementine } \times \\
\text { Orlando) }(3850)\end{array}$ & 'Robinson' mandarin & Aur/Citreae/Citrinae & 7 \\
\hline 84 & Citrus sinensis (L.) Osbeck (3858) & 'Pineapple' sweet orange & Aur/Citreae/Citrinae & 7 \\
\hline 85 & Citrus sunki hort. ex Tan. (3143) & 'Sunki' mandarin & Aur/Citreae/Citrinae & 7 \\
\hline 86 & Citrus aurantium L. (3289) & Sour orange var. salicifolia & Aur/Citreae/Citrinae & 8 \\
\hline 87 & Citrus nobilis Lour. (3845) & 'King' mandarin & Aur/Citreae/Citrinae & 8 \\
\hline 88 & Citrus reticulata Blanco (300) & 'Parson's Special' mandarin & Aur/Citreae/Citrinae & 8 \\
\hline 89 & Citrus reticulata Blanco (3812) & Unnamed mandarin & Aur/Citreae/Citrinae & 8 \\
\hline 90 & $\begin{array}{l}\text { Citrus reticulata hybrid (Clementine } \times \\
\text { Orlando) }(3851)\end{array}$ & 'Lee' mandarin & Aur/Citreae/Citrinae & 8 \\
\hline 91 & Afraegle paniculata (Schum.) Engl. & Nigerian Powder flask & & $*$ \\
\hline 92 & Atalantia ceylanica (Am.) Oliv. (3725) & Atalantia & Aur/Citreae/Citrinae & $*$ \\
\hline 93 & Citropsis daweana Swing. \& M. Kell (3294) & Citropsis & Aur/Citreae/Citrinae & $*$ \\
\hline 94 & Citrus reticulata Blanco (3991) & 'USDA 88-2' mandarin & Aur/Citreae/Citrinae & * \\
\hline 95 & $\begin{array}{l}\text { Clausena harmandiana Pierre ex Guillaumin } \\
\text { (4034) }\end{array}$ & Clausena & Aur/Clauseneae/Clauseninae & $*$ \\
\hline 96 & Fortunella hindsii Champ. ex Benth. (3789) & Unnamed kumquat & Aur/Citreae/Citrinae & $*$ \\
\hline 97 & Severinia buxifolia (Poiret) Tan. (1495) & Chinese box orange & Aur/Citreae/Citrinae & $*$ \\
\hline 98 & Severinia buxifolia (Poiret) Tan. (3724) & Chinese box orange & Aur/Citreae/Citrinae & $*$ \\
\hline
\end{tabular}


plant-damaging freezes occurred in the first two years. For certain taxa, data were obtained from only a few plants, making the disease category determinations challenging. These are indicated in the tables as insufficient data in the disease category column. Table 1 has a list of accessions included in the field trial, their scientific and common names, taxonomical groups and assigned HLB disease categories. The complete qPCR data indicating $\mathrm{Ct}$ values of all test plants over a period of six years is shown in data tables.

Response of accessions to HLB exposure by disease category. Category 1. Two accessions, Clausena excavata (pink wampee) and Glycosmis pentaphylla (orange berry) were considered immune.
Despite repeated testing of multiple samples for a period of six years, we were never able to detect ' $\mathrm{Ca}$. L. asiaticus' in either accession.

Category 2. Six accessions were considered resistant: Bergera koenigii (curry leaf), Casimiroa edulis (white sapote), Eremocitrus glauca (Australian desert lime), Murraya paniculata ('Orange jasmine', which was recently classified as Murraya exotica [A. Beattie, personal communication]), Naringi crenulata (Hesperethusa), and Zanthoxylum ailanthoides (Japanese prickly ash). Even though taxa assigned to this group were able to support replication of the pathogen transiently, the liberibacter bacterium could not establish in these hosts, although a low level of replication of the bacterium was

Table 2. Huanglongbing disease category based on pathogen titers, as estimated by annual real time polymerase chain reaction results (cycle threshold [Ct] values) ${ }^{\mathrm{a}}$

Ct range for 'Candidatus $L$. asiaticus'

(number of plants) ${ }^{\mathbf{c}}$

\begin{tabular}{|c|c|c|c|c|c|c|c|c|}
\hline No. & Common name of taxa (CRC number $)^{\mathrm{a}}$ & Categoryb $^{\mathbf{b}}$ & Year 1 & Year 2 & Year 3 & Year 4 & Year 6 & $\begin{array}{l}\text { Aslan citrus psyl } \\
\text { colonization }^{\mathbf{d}}\end{array}$ \\
\hline 1 & Pink wampee (3166) & 1 & $40(8)$ & $40(7)$ & $40(6)$ & $40(6)$ & $40(6)$ & $0.56 / 0.94$ \\
\hline 2 & Orange berry (3285) & 1 & $40(8)$ & $40(8)$ & $40(8)$ & $40(8)$ & $40(8)$ & $0.16 / 0.03$ \\
\hline 3 & Curry leaf $(3165)(\mathrm{P})$ & 2 & $40(8)$ & $40(7)$ & $36-40(7)$ & $40(7)$ & $40(7)$ & $1.35 / 1.81$ \\
\hline 4 & White sapote & 2 & $31-40(8)$ & $37-40(8)$ & $40(8)$ & $40(8)$ & $39-40(7)$ & $0.00 / 0.00$ \\
\hline 5 & Australian desert lime hybrid (4105) (M) & 2 & $36-40(8)$ & $35-40(7)$ & $40(7)$ & $40(7)$ & $35-40(5)$ & $0.20 / 0.90$ \\
\hline 6 & 'Orange jasmine' (1637) & 2 & $38-40(8)$ & $35-40(5)$ & $40(5)$ & $40(5)$ & $40(5)$ & $1.07 / 1.48$ \\
\hline 7 & Hesperethusa (2879) & $2 *$ & $37-40(7)$ & $40(2)$ & $36-40(2)$ & Dead & Dead & NA \\
\hline 8 & Japanese prickly ash & 2 & $40(7)$ & $40(7)$ & $40(5)$ & $40(3)$ & $39-40(2)$ & $0.29 / 0.00$ \\
\hline 9 & 'Mountain' citron (3780) & 3 & $38-40(8)$ & $38-40(4)$ & $29-40$ (4) & $28-40(2)$ & $40(1)$ & $0.27 / 0.64$ \\
\hline 10 & $\begin{array}{l}\text { Australian finger lime var. 'Sanguinea' } \\
(1484)(\mathrm{PM})\end{array}$ & 3 & $30-40(6)$ & $30-40(6)$ & $36-40(5)$ & $31-40(5)$ & $30-40(5)$ & $0.24 / 0.48$ \\
\hline 11 & 'Sydney hybrid' (1485) (PM) & 3 & $27-40(8)$ & $30-40(8)$ & $28-40(8)$ & $33-40(7)$ & $29-37(6)$ & $0.26 / 1.68$ \\
\hline 12 & Large-leaf Australian wild lime (3785) (PM) & $3^{*}$ & $34-40(6)$ & $37(2)$ & $30(1)$ & Dead & & $0.14 / 0.86$ \\
\hline 13 & 'Faustrimedin' (1466) & 3 & $37-40(8)$ & $25-40(6)$ & $24-40(4)$ & $40(1)$ & $40(1)$ & $0.27 / 0.65$ \\
\hline 14 & 'Simmon's trifoliate' (3549) (PP) & 3 & $40(8)$ & $32-40(8)$ & $35-40(7)$ & $28-40(7)$ & $40(6)$ & $0.07 / 0.04$ \\
\hline 15 & Indian bael fruit $(\mathrm{P})$ & 4 & $27-40(5)$ & $27-32(4)$ & $31-34(4)$ & $33-37(4)$ & $36-40(4)$ & $0.27 / 0.50$ \\
\hline 16 & Chevalier's Aeglopsis (2878) & 4 & $40(3)$ & $28-40(3)$ & $39-40(3)$ & $40(1)$ & $40(1)$ & NA \\
\hline 17 & Uganda powder flask (3514) & 4 & $28-40(8)$ & $30-40(7)$ & $31-40(6)$ & $40(6)$ & $40(6)$ & $0.35 / 0.62$ \\
\hline 18 & 'Khasi' papeda (3052) & 4 & $30-40(8)$ & $24-40(7)$ & $23-40(6)$ & $31-40(6)$ & $40(6)$ & $0.42 / 1.04$ \\
\hline 19 & Australian round lime (3673) & 4 & $26-40(8)$ & $28-40(8)$ & $29-40(7)$ & $31-40(6)$ & $30-40(6)$ & $0.38 / 0.92$ \\
\hline 20 & 'Hawaiian mock orange'(3171) & 4 & $28-40(8)$ & $35-40(8)$ & $35-40(8)$ & $40(8)$ & $40(7)$ & $0.70 / 1.20$ \\
\hline 21 & 'Little-leaf' trifoliate (4007) (P) & 5 & $32-40(8)$ & $29-40(8)$ & $26-40(8)$ & $28-40(8)$ & $28-40(8)$ & $0.06 / 0.03$ \\
\hline 22 & 'X639' trifoliate hybrid (3957) (P) & 5 & $32-40(8)$ & $30-40(8)$ & $25-40(8)$ & $27-36(8)$ & $27-34(8)$ & $0.39 / 0.77$ \\
\hline 23 & 'Nasnaran' mandarin (2485) (P) & 6 & $22-40(8)$ & $22-27(8)$ & $23-27(7)$ & $27-29(7)$ & $26-31(7)$ & $0.62 / 1.28$ \\
\hline 24 & 'India' lime (2450) (P) & 6 & $22-40(8)$ & $23-30(7)$ & $22-28(7)$ & $26-31(7)$ & $26-33(7)$ & $0.83 / 1.79$ \\
\hline 25 & 'Olivelands' sour orange (2717) $(\mathrm{PP})$ & 6 & $38-40(8)$ & $23-29(8)$ & $24-29(8)$ & $25-29(8)$ & $28-33(8)$ & $0.76 / 1.72$ \\
\hline 26 & 'Standard' sour orange $(628)(\mathrm{P})$ & 6 & $26-40(8)$ & $22-28(8)$ & $22-28(8)$ & $24-30(8)$ & $28-35(8)$ & $0.29 / 1.14$ \\
\hline 27 & 'Davao' lemon (2427) & 6 & $25-40(8)$ & $25-32(7)$ & $23-31(7)$ & $27-32(7)$ & $23-33(7)$ & $0.78 / 1.70$ \\
\hline 28 & 'Limon Real' papeda (2317) & 6 & $21-40(8)$ & $26-34(8)$ & $26-31(8)$ & $27-32(8)$ & $29-35(8)$ & $0.62 / 1.58$ \\
\hline 29 & 'Hassaku pummelo' hybrid (3907) & 6 & $20-40(8)$ & $21-33(8)$ & $24-40(8)$ & $23-36(7)$ & $21-40(6)$ & $0.54 / 1.77$ \\
\hline 30 & 'Hassaku pummelo' hybrid (3942) & 6 & $20-40(8)$ & $21-31(8)$ & $23-31(8)$ & $24-35(7)$ & $25-35(7)$ & $0.73 / 1.83$ \\
\hline 31 & Ex-India sour orange hybrid (3715) & 6 & $20-40(8)$ & $23-26(6)$ & $25-40(4)$ & $26-29(3)$ & $26-31(3)$ & $0.76 / 1.36$ \\
\hline 32 & 'Florida' rough lemon $(400)(\mathrm{P})$ & 6 & $26-40(8)$ & $23-37(8)$ & $24-28(7)$ & $25-29(6)$ & $27-31(6)$ & $0.53 / 1.37$ \\
\hline 33 & 'Koji' mandarin (3147) & 6 & $21-40(8)$ & $25-40(7)$ & $21-27(6)$ & $25-31(6)$ & $24-29(6)$ & $0.25 / 0.71$ \\
\hline 34 & 'Palestine' sweet lime (1482) (P) & 6 & $23-40(8)$ & $26-40(8)$ & $24-28(7)$ & $25-29(5)$ & $28-30(5)$ & $0.75 / 1.88$ \\
\hline 35 & 'Frost Nucellar Eureka' lemon (3005) (P) & 6 & $22-40(7)$ & $28-40(7)$ & $26-31(7)$ & $27-29(4)$ & $28-35(4)$ & $0.77 / 1.38$ \\
\hline 36 & 'Interdonato' lemon (3593) & 6 & $22-40(8)$ & $24-30(6)$ & $26-33(6)$ & $28-31(6)$ & $27-30(6)$ & $0.59 / 1.66$ \\
\hline 37 & Iranian lemon (3885) (PP) & 6 & $22-28(8)$ & $22-32(7)$ & $23-31(7)$ & $26-31(6)$ & $27-34(6)$ & $0.85 / 1.81$ \\
\hline 38 & 'Lamas' lemon (3919) (PP) & 6 & $22-40(8)$ & $28-40(5)$ & $25-33(5)$ & $29-30(4)$ & $28-34(4)$ & $0.61 / 1.89$ \\
\hline 39 & 'Santa Barbara' red lime (712) $(\mathrm{P})$ & 6 & $21-34(8)$ & $25-35(8)$ & $26-30(8)$ & $28-31(8)$ & $29-33(8)$ & $0.81 / 1.94$ \\
\hline 40 & 'Winged' lime (2320) & 6 & $31-40(8)$ & $21-40(7)$ & $22-32(6)$ & $27-31(4)$ & $29-33(4)$ & $0.37 / 1.04$ \\
\hline 41 & 'Alemow' hybrid (3842) (P) & 6 & $21-40(8)$ & $27-40(8)$ & $28-40(8)$ & $28-39(8)$ & $31-34(8)$ & $0.83 / 1.72$ \\
\hline 42 & 'Kao Pan' pummelo (2242) (M) & 6 & $23-40(8)$ & $19-24(8)$ & $21-34(7)$ & $28-31(7)$ & $22-40(7)$ & $0.56 / 1.67$ \\
\hline 43 & 'Kao Panne' pummelo (2248) (M) & 6 & $31-40(8)$ & $22-40(7)$ & $22-30(7)$ & $24-29(7)$ & $24-35(7)$ & $0.61 / 1.75$ \\
\hline 44 & 'Reinking' pummelo (3805) (M) & 6 & $22-40(8)$ & $23-31(6)$ & $20-40(6)$ & $22-30(4)$ & $24-32(4)$ & $0.57 / 1.36$ \\
\hline 45 & 'Diamante' citron (3523) (M) & 6 & $20-40(8)$ & $26-32(8)$ & $23-33(8)$ & $27-34(8)$ & $28-34(8)$ & $0.97 / 1.81$ \\
\hline 46 & 'South Coast Field Station' citron (3546) (M) & 6 & $21-30(8)$ & $25-35(8)$ & $20-33(7)$ & $26-32(5)$ & $31-32(5)$ & $0.86 / 2.07$ \\
\hline
\end{tabular}

(continued on next page)

a $(\mathrm{M})=$ Monoembryonic $;(\mathrm{PM})=$ presumed to be monoembryonic $;(\mathrm{P})=$ polyembryonic; $(\mathrm{PP})=$ presumed to be polyembryonic.

b Asterisks $(*)$ indicate insufficient data for disease rating.

c Range of $\mathrm{Ct}$ values for five years indicated. Numbers in parentheses after the $\mathrm{Ct}$ values indicate number of living plants at each time point from which data were recorded. Psyllid colonization data are from Westbrook et al. 2011.

${ }^{\mathrm{d}}$ Mean rating of adults/nymphs was determined on a scale of 0 to 3 (for adults: $0=$ no psyllids, $1=1$ to $5,2=6$ to $15,3=>15$; for nymphs: $0=$ no nymphs, $1=1$ to $10,2=11$ to $30,3=>30)$. NA $=$ not available. 
detected periodically. Death of many plants in the resistant category was attributed to cold weather during the early part of the field trial (2009 and 2010 winters) and not because of HLB disease issues (Inch et al. 2014).

Bergera koenigii (curry leaf) is a commonly cultivated plant and is well known to be a preferred host for the psyllid (Damsteegt et al. 2010; Westbrook et al. 2011) (Table 2). In the current field trial, we detected the HLB pathogen in curry leaf only once in six years. White sapote, cultivated for its edible fruit, was mostly pathogenfree. Australian desert lime was resistant with a transient, low level of ' $\mathrm{Ca}$. L. asiaticus' replication (five samples had $\mathrm{Ct}$ values ranging from 35 to 39). In the current field trial, 'Orange jasmine' was considered to be a resistant plant, even though ' $\mathrm{Ca}$. L. asiaticus' $\mathrm{Ct}$ values of 35 to 38 were recorded in six of 28 data points. The overall appearance of 'Orange jasmine' was that of a healthy tree with generally lush green foliage. The pathogen could be detected from symptomatic leaves showing slight blotchy mottle and yellowing of the foliage. However, the pathogen never established and the trees were regarded as healthy in the experimental field with high incidence of HLB. Most trees of the tropical species Hesperethusa died in the first two years, probably due to environmental factors. Ct values of 37 to 38 were recorded from four trees in years 1 to 3 , indicating brief

Table 2. (continued from preceding page)

\begin{tabular}{|c|c|c|c|c|c|c|c|c|}
\hline \multirow[b]{2}{*}{ No. } & \multirow[b]{2}{*}{ Common name of taxa (CRC number $)^{a}$} & \multirow[b]{2}{*}{ Category $^{\mathbf{b}}$} & \multicolumn{5}{|c|}{$\begin{array}{c}\text { Ct range for 'Candidatus } \mathbf{L} \text {. asiaticus' } \\
\text { (number of plants) }\end{array}$} & \multirow{2}{*}{$\begin{array}{l}\text { Asian citrus psyllid } \\
\text { colonization }^{\mathbf{d}}\end{array}$} \\
\hline & & & Year 1 & Year 2 & Year 3 & Year 4 & Year 6 & \\
\hline 47 & 'Indian' citron hybrid (661) & 6 & $23-40(8)$ & $26-37(8)$ & $29-36(8)$ & $24-35(8)$ & $30-36(7)$ & $0.73 / 1.54$ \\
\hline 48 & 'Kinkoji Unshiu' (3816) (PP) & 6 & $26-40(8)$ & $22-27(8)$ & $22-25(8)$ & $26-28(7)$ & $25-33(7)$ & $0.68 / 1.75$ \\
\hline 49 & 'Konejime' sour orange hybrid (3611) (PP) & 6 & $20-40(8)$ & $22-28(8)$ & $23-29(8)$ & $25-28(6)$ & $27-30(6)$ & $0.42 / 1.54$ \\
\hline 50 & 'Fremont' mandarin (3558) & 6 & $21-40(8)$ & $21-26(8)$ & $20-24(6)$ & $26-27(3)$ & $26-28(3)$ & $0.76 / 1.40$ \\
\hline 51 & Unnamed lemon hybrid (3172) & 6 & $25-40(8)$ & $26-35(7)$ & $26-31(7)$ & $26-32(7)$ & $23-32(7)$ & $0.72 / 1.28$ \\
\hline 52 & 'Nasho Daidai' sour orange (2588) (P) & 6 & $27-40(8)$ & $21-28(7)$ & $23-27(7)$ & $26-34(7)$ & $26-40(7)$ & $0.48 / 2.07$ \\
\hline 53 & 'Volkamer' lemon hybrid (3050) (P) & 6 & $19-32(7)$ & $22-28(7)$ & $25-29(7)$ & $24-30(6)$ & $28-37(6)$ & $0.73 / 1.69$ \\
\hline 54 & 'Kalpi’ papeda (1455) & 6 & $27-40(8)$ & $27-40(7)$ & $28-30(4)$ & $29-32(4)$ & $31-34(4)$ & $0.45 / 1.66$ \\
\hline 55 & Chinese Box orange (1497) (M) & 6 & $28-40(8)$ & $26-40(8)$ & $23-33(8)$ & $26-31(8)$ & $26-40(8)$ & $0.52 / 0.48$ \\
\hline 56 & Chinese Box orange (4107) (M) & 6 & $36-40(5)$ & $39-40(4)$ & 20-30 (4) & $26-31(4)$ & $28(4)$ & NA \\
\hline 57 & Chinese Box orange (1491) (M) & 6 & $39-40$ (4) & $31-40(4)$ & $27-36(4)$ & $29-30(3)$ & $30-40(3)$ & NA \\
\hline 58 & 'Rusk' citrange (301) (P) & 6 & $32-40(8)$ & $21-40(8)$ & $22-28(8)$ & $25-27(8)$ & $27-29(8)$ & $0.43 / 1.27$ \\
\hline 59 & 'S-281' citrangelo (3552) & 6 & $28-40(8)$ & $25-40(8)$ & $25-36(7)$ & $26-32(3)$ & $29-33(3)$ & $0.66 / 1.55$ \\
\hline 60 & 'Swingle' citrumelo (3771) $(\mathrm{P})$ & 6 & $28-40(8)$ & $22-40(8)$ & $23-26(8)$ & $24-29(8)$ & $29-33(8)$ & $0.56 / 2.09$ \\
\hline 61 & 'Mexican' lime (3822) (P) & 7 & $22-40(7)$ & $28-30(3)$ & $26-28(3)$ & $24-29(3)$ & 24-31 (3) & $0.57 / 1.93$ \\
\hline 62 & 'Goutoucheng' sour orange (3929) (PP) & 7 & $23-40(8)$ & $23-30(8)$ & $23-28(8)$ & $22-30(7)$ & $24-32(7)$ & $0.70 / 1.30$ \\
\hline 63 & 'Zhuluan' sour orange hybrid (3930) (PP) & 7 & $21-40(7)$ & $22-40(7)$ & $22-29(5)$ & $24-30(5)$ & $26-33(5)$ & $0.48 / 1.48$ \\
\hline 64 & Unnamed tangor (3149) & 7 & $20-40(8)$ & $22-37(4)$ & $24-27(4)$ & $25-28(3)$ & $25-29(3)$ & $0.54 / 1.04$ \\
\hline 65 & 'Yama-mikan' sour orange (3474) & 7 & $32-40(7)$ & $21-28(7)$ & $22-30(6)$ & $25-30(5)$ & $27-31(5)$ & $0.52 / 1.15$ \\
\hline 66 & 'Frost Nucellar Lisbon' lemon (3176) (P) & 7 & $23-40(8)$ & $27-40(7)$ & $27-30(6)$ & $29(3)$ & $29-32(3)$ & $0.81 / 1.73$ \\
\hline 67 & ‘Mesero’ lemon (3892) & 7 & $24-40(8)$ & $28-40(8)$ & $28-40(6)$ & $29-31(2)$ & $33(2)$ & $0.63 / 1.41$ \\
\hline 68 & Kokni orange (3564) $(\mathrm{P})$ & 7 & $23-40(8)$ & $25-35(7)$ & $24-29(4)$ & $30(1)$ & $34(1)$ & $0.46 / 1.00$ \\
\hline 69 & 'Kitchli' sour orange (3225) & $7 *$ & $36-40(7)$ & $36(1)$ & Dead & & & NA \\
\hline 70 & 'Mato Buntan’ pummelo (3945) (M) & 7 & $21-40(7)$ & $22-36(7)$ & $23-40(5)$ & $25-27(2)$ & $26-31(2)$ & $0.90 / 1.38$ \\
\hline 71 & ‘Egami Buntan’ pummelo (3959) (M) & 7 & $22-40(8)$ & $21-40(6)$ & $22-27(5)$ & $26-31(5)$ & $23-40(5)$ & $0.50 / 1.60$ \\
\hline 72 & 'Pomelit' pummelo hybrid (4026) & 7 & $21-40(8)$ & $22-27(8)$ & $22-29(7)$ & $25-30(6)$ & $27-34(6)$ & $0.55 / 1.72$ \\
\hline 73 & $\begin{array}{l}\text { 'Tahitian' pummelo } \times \text { 'Star Ruby' grapefruit } \\
\text { (3781) }\end{array}$ & 7 & $20-40(8)$ & $22-30(7)$ & $24-40(6)$ & $27-29(2)$ & $28-30(2)$ & $0.63 / 1.89$ \\
\hline 74 & 'Dweet' tangor $(3018)(\mathrm{P})$ & 7 & $28-40(8)$ & $23-40(8)$ & $22-28(5)$ & $26-28(4)$ & $25-27(3)$ & $0.47 / 1.27$ \\
\hline 75 & 'Tien Chieh' mandarin (2590) & 7 & $40(8)$ & $20-24(8)$ & $19-23(6)$ & $24-27(2)$ & $23-24(2)$ & $1.11 / 1.63$ \\
\hline 76 & 'Frua' mandarin $(3022)(\mathrm{P})$ & 7 & $21-40(5)$ & $21-28(2)$ & $21(1)$ & Dead & & $0.73 / 1.27$ \\
\hline 77 & 'Soh Niamtra' mandarin (3260) & 7 & $19-40(8)$ & $20-24(7)$ & $20-24(4)$ & $23-24(3)$ & $22-26(3)$ & $0.87 / 1.65$ \\
\hline 78 & 'Scarlett Emperor' mandarin (3326) & 7 & $19-40(8)$ & $23-33(5)$ & $23-27$ (4) & $25-28(4)$ & $27-30(4)$ & $0.56 / 0.96$ \\
\hline 79 & 'Belady' mandarin (3363) & 7 & $34-40(8)$ & $21-39(7)$ & $19-28(6)$ & $20-26(4)$ & $24-40(3)$ & $0.52 / 1.23$ \\
\hline 80 & 'Som Keowan’ mandarin (3752) & 7 & $21-40(8)$ & $21-26(8)$ & $22-27(5)$ & $25-27(4)$ & $26-34(4)$ & $0.75 / 1.57$ \\
\hline 81 & 'Koster’ mandarin (3958) & 7 & $19-40(8)$ & $24-40(6)$ & $20-40(5)$ & $30(1)$ & $37(1)$ & $0.69 / 1.72$ \\
\hline 82 & 'Sun Chu Sha' mandarin (4003) (P) & 7 & $33-40(8)$ & $20-25(8)$ & $21-26(7)$ & $23-25(4)$ & $22-27(4)$ & $0.55 / 1.62$ \\
\hline 83 & 'Robinson' mandarin (3850) (M) & 7 & $25-40(8)$ & $20-40(8)$ & $20-25(5)$ & $24-25(4)$ & $24-27(4)$ & $0.55 / 0.90$ \\
\hline 84 & 'Pineapple' sweet orange (3858) (P) & 7 & $24-40(8)$ & $22-35(7)$ & $23-26(5)$ & $23-27(4)$ & $30-38(2)$ & $0.39 / 1.21$ \\
\hline 85 & ‘Sunki’ mandarin (3143) (P) & 7 & $26-40(8)$ & $23-37(7)$ & $24-27(3)$ & $26(2)$ & $27-29(2)$ & $0.80 / 1.37$ \\
\hline 86 & Sour orange var. salicifolia (3289) (PP) & 8 & $34-40(3)$ & $24-28(2)$ & $23-25(2)$ & $28(1)$ & $32(1)$ & $0.43 / 1.00$ \\
\hline 87 & 'King' mandarin $(3845)(\mathrm{P})$ & 8 & $39-40(8)$ & $19-40(4)$ & $21(1)$ & Dead & & $0.52 / 1.11$ \\
\hline 88 & 'Parson's Special' mandarin (300) & 8 & $22-40(8)$ & $21-40(5)$ & $21-23(2)$ & Dead & & $0.54 / 0.93$ \\
\hline 89 & Unnamed mandarin (3812) & 8 & $23-40(8)$ & $22-26(5)$ & $22-30(3)$ & $26(1)$ & $26(1)$ & $0.52 / 1.00$ \\
\hline 90 & 'Lee' mandarin (3851) (M) & 8 & $21-40(7)$ & $19-40(5)$ & $22-23(2)$ & $26(1)$ & $25(1)$ & $0.43 / 1.04$ \\
\hline 91 & Nigerian Powder flask & $*$ & $38-40(8)$ & Dead & & & & $0.55 / 1.26$ \\
\hline 92 & Atalantia (3725) & $*$ & Dead & & & & & NA \\
\hline 93 & Citropsis (3294) & $*$ & $40(3)$ & Dead & & & & NA \\
\hline 94 & USDA 88-2 (3991) & $*$ & Dead & & & & & NA \\
\hline 95 & Clausena harmandiana (4034) & $*$ & $40(7)$ & $35(1)$ & Dead & & & $0.19 / 0.15$ \\
\hline 96 & Unnamed kumquat(3789) (P) & $*$ & Dead & & & & & NA \\
\hline 97 & Chinese box orange (1495) (M) & $*$ & $38-40(2)$ & Dead & & & & NA \\
\hline 98 & Chinese box orange (3724) (M) & $*$ & Dead & & & & & NA \\
\hline
\end{tabular}


periods of pathogen replication. In Japanese prickly ash, belonging to the subfamily Toddalioideae, one sample had a $\mathrm{Ct}$ value of 39 (out of a total of 24 samples).

Category 3. Accessions in this category consisted of HLB tolerant taxa that showed different disease responses within the eight test plants belonging to one accession, probably due to seedling variations (Table 2). Citrus halimii ('Mountain' citron), Microcitrus australasica (Australian finger lime), Microcitrus hybrid ('Sydney hybrid'), Microcitrus inodora (Large-leaf Australian wild lime), Microcitronella sp. ('Faustrimedin'), and Poncirus trifoliata ('Simmon's trifoliate') were grouped in this category. Most accessions in this group are presumed to be monoembryonic. It is conceivable that the zygotic seedlings may have genetic differences that may result in nonuniform disease response phenotypes in the eight plants of one accession tested. In addition, the progeny of known hybrids ('Sydney hybrid' and 'Faustrimedin') showed segregation of characters. 'Simmon's trifoliate' is assumed to be polyembryonic (M. L. Roose, unpublished information) and also is reported to be a nonpreferred host for the psyllid (Westbrook et al. 2011). Under field conditions, several other factors, such as freeze damage, canker, and other biotic and abiotic causes, also played a role in the death of plants. We determined the probable cause of death of individual plants on a case-by-case basis, mainly depending on apparent disease status before a plant died.

The 'Mountain' citron did not perform well in the Fort Pierce field, since four of the eight plants died after year 1, probably due to environmental factors. Two of the surviving plants died during years 4 and 5, presumably because of HLB, since Ct values of 27 to 28 were recorded from the samples collected and symptoms of HLB were observed. However, two plants remained healthy and free of the pathogen for four years and appeared HLB resistant. Four accessions of Microcitrus spp. (two known hybrids) included in the field trial were considered as tolerant plants belonging to category 3 . The genus Microcitrus is presumed to be monoembryonic and seedling genetic variations may exist. The plants had excellent field tolerance and none appeared to die because of HLB. Symptoms of HLB that typically occur in citrus were not observed in Microcitrus spp. In the tolerant accession 'Simmon's trifoliate', two plants died due to HLB and the other six appeared resistant. Psyllids tend to avoid colonizing cultivars of Poncirus trifoliata, resulting in plants being exposed to less inoculum (Richardson and Hall 2013; Hall et al. 2015).

Category 4. This group of plants are considered HLB tolerant, since they show recovery from ' $\mathrm{Ca}$. L. asiaticus' infections. One citrus accession and five citrus relative genera were included in category 4: Aegle marmelos (Indian bael), Aeglopsis chevalieri (Chevalier's Aeglopsis), Balsamocitrus daweii (Uganda Powder flask), Citrus latipes ('Khasi' papeda), Microcitrus australis (Australian round lime), and Murraya paniculata ('Hawaiian mock orange').

Indian bael is a plant of religious significance important to certain ethnic groups. In the field trial, three of the seven Indian bael trees planted died in year 1 due to freeze damage. One plant died in year 2, probably due to HLB, since a Ct value of 27 was recorded. Indian bael had disease symptoms on the branches that tested positive by qPCR. The other four plants had $\mathrm{Ct}$ values of 27 to 37 at various times. During year 6, three plants appeared to be free of ' $\mathrm{Ca}$. L. asiaticus' and one tree had a Ct of 36 for ' $\mathrm{Ca}$. L. asiaticus'. Five trees of Chevalier's Aeglopsis died during the first winter, presumably because of the cold weather. One surviving plant remained resistant for six years, and the pathogen was never detected from this plant. Two plants had $\mathrm{Ct}$ values of 28 in year 2 but, in the subsequent year, the pathogen could not be detected despite extensive sampling and testing. Chevalier's Aeglopsis lacked typical HLB symptoms. However, due to other factors seemingly not related to HLB, both these plants died during year 4 of the study. Uganda powder flask also appeared to recover from infections. Two plants died by year 3, one apparently because of HLB and another died for other reasons. As observed in many other noncitrus genera, the ' $\mathrm{Ca}$. L. asiaticus'positive plants of Uganda powder flask did not have identifiable HLB symptoms. In six other plants, Ct values of 28 to 35 were recorded in years 1, 2, and 3; during years 4 and 6 , all living plants of this accession were free of ' $\mathrm{Ca}$. L. asiaticus'. Australian round lime showed excellent field tolerance similar to other accessions of Microcitrus spp. that were evaluated in this field trial. Most of the trees were tolerant and symptom-free in spite of having $\mathrm{Ct}$ values of 30 to 38 for ' $\mathrm{Ca}$. L. asiaticus'. 'Hawaiian mock orange' was considered as an HLB-tolerant accession belonging to category $4 . \mathrm{Ct}$ values of 28 to 38 were detected in most plants of 'Hawaiian mock orange' (in six of 28 data points) but, with time, the pathogen was eliminated from the plant. One citrus accession, 'Khasi' papeda, was included in category 4. Four of the eight 'Khasi' papeda plants had ' $C a$. L. asiaticus' Ct values of 24 to 34 during years 1 to 4 . However, in year 6, the six surviving plants appeared healthy and had no detectable HLB pathogen, indicating recovery from infections.

Category 5. Only two accessions were grouped under HLBtolerant category 5 , characterized by a delay in ' $\mathrm{Ca}$. L. asiaticus' infections. Many plants of Poncirus trifoliata ('Little-leaf' trifoliate) and XCitroncirus hybrid ('X639' trifoliate) had delayed infections. Blotchy mottle leaf symptoms were present in ' $\mathrm{Ca}$. L. asiaticus'positive plants in this group.

Category 6. Categories 6, 7, and 8 were all basically susceptible to ' $C a$. L. asiaticus', with typical HLB symptoms. Category 6 consisted of 38 accessions, mostly belonging to the genera Citrus, Severinia, and XCitroncirus. The plants were clearly susceptible (based on ' $C a$. L. asiaticus' titers and disease symptoms) but most of the accessions had all eight plants living for at least four years and the plants retained their leaves, in spite of having disease symptoms and high pathogen titers. Chinese box orange also had classic symptoms of HLB and diseased plants showed stunted growth. HLB in Chinese box orange has been documented earlier (Hung et al. 2001; Hu et al. 2014). In this field trial, among the citrus accessions tested, most of the citrons, limes, and lemons belonged to category 6 and seem to perform better than other citrus types. Certain mandarins, pummelos, and sour oranges also belonged to category 6 (Table 1).

Category 7. In this group, we included accessions that lived for up to four years but had severe disease symptoms including loss of foliage. About 25 accessions, including many mandarins, certain pummelos, sour oranges, some lemons, and limes, were in category 7.

Category 8. Plants in this susceptible group mostly died within the first four years of the field trial. Certain mandarins were grouped under category 8 . Table 2 shows the range of ' $\mathrm{Ca}$. L. asiaticus' $\mathrm{Ct}$ values for all accessions during the six-year field trial.

We had eight accessions that could not be grouped under any category because of insufficient data. Table 3 shows Ct values of representative samples from each disease category. Table 4 shows disease categories observed in the different genera included in the field trial.

HLB disease scores associated with ACP abundance and colonization. In a longitudinal study conducted in Fort Pierce, FL, involving the exact same test plants, Westbrook et al. (2011) studied the colonization of ACP. We have compared the HLB categories and ' $\mathrm{Ca}$. L. asiaticus' titers assigned in the present study with ACP abundance and colonization data (Westbrook et al. 2011) (Table 2). It has been shown that native rutaceous plants that serve as hosts for the psyllid can affect the epidemiology of HLB and noninfection of certain taxa may be due to the inability of the psyllid to transmit the HLB pathogen (Sétamou et al. 2016).

Plants in category 1 had either medium or low scores of ACP infestation. In category 2, Casimiroa edulis (White sapote) was reported as a nonhost for ACP, with a score of 0 for both adult and nymph infestations (Halbert and Manjunath 2004; Westbrook et al. 2011). However, we have detected a transient presence of ' $\mathrm{Ca}$. L. asiaticus', indicating that occasional psyllid feeding may occur. Zanthoxylum ailanthoides (Japanese prickly ash) had a low score of 0.29 for adult ACP infestations and did not support colonization (Westbrook et al. 2011). Most species of genus Zanthoxylum are reported to be poor hosts for the ACP (Halbert and Manjunath 2004). The Australian accession Eremocitrus glauca (Australian desert lime) was not a preferred host for the psyllid, since it had low scores of 0.2 and 0.9 for adult and nymph infestations, respectively (Westbrook et al. 2011). In contrast, Murraya paniculata ('Orange jasmine') and 
Bergera koenigii (curry leaf) are known to be preferred hosts for ACP (Damsteegt et al. 2010; Tsai et al. 2002; Westbrook et al. 2011).

In category 3, with variations observed in seedling populations, Poncirus trifoliata ('Simmon's trifoliate') had very poor scores for psyllid infestation and colonization (Westbrook et al. 2011). Other accessions in this category had psyllid infestation numbers ranging from 0.14 in Large-leaf Australian lime to 0.27 in 'Faustrimedin'. Colonization numbers ranged from 0.48 in Australian finger lime to 1.68 in 'Sydney hybrid'. In general, psyllid colonization levels were similar to other HLB-sensitive accessions. Most accessions that we grouped in category 4 had moderate psyllid ratings. The score ranged from 0.27 to 0.70 for adults (in Indian bael and 'Hawaiian mock orange', respectively) and 0.5 to 1.77 for nymphs. 'Hawaiian mock orange', despite being favorable for psyllid colonization, still appears to have tolerance to HLB. In category 5, consisting of HLB tolerant plants showing delayed ' $\mathrm{Ca}$. L . asiaticus' infections, only two accessions were included. 'Little-leaf' trifoliate is not a

Table 3. Representative taxa from each of the eight disease categories and yearly levels of the huanglongbing (HLB) pathogen ${ }^{\mathrm{a}}$

\begin{tabular}{|c|c|c|c|c|c|}
\hline $\begin{array}{l}\text { Name of the taxa } \\
\text { (CRC number) }\end{array}$ & Year 1 & Year 2 & Year 3 & Year 4 & Year \\
\hline \multicolumn{6}{|c|}{ Category 1 (immune: no pathogen detected) } \\
\hline Glycosmis pentaphylla & 40 & 40 & 40 & 40 & 40 \\
\hline \multirow[t]{3}{*}{ Orange berry (3285) } & 40 & 40 & 40 & 40 & 40 \\
\hline & 40 & 40 & 40 & 40 & 40 \\
\hline & 40 & 40 & 40 & 40 & 40 \\
\hline \multicolumn{6}{|c|}{ Category 2 (resistant: transient replication) } \\
\hline Eremocitrus glauca hybrid & 38 & 40 & 40 & 40 & 36 \\
\hline \multirow{3}{*}{$\begin{array}{l}\text { Australian desert lime } \\
(4105)\end{array}$} & 39 & 40 & 40 & 40 & $\mathrm{D}^{*}$ \\
\hline & 36 & 35 & 40 & 40 & 40 \\
\hline & 40 & 40 & 40 & 40 & 40 \\
\hline \multicolumn{6}{|c|}{ Category 3 (tolerant: seedling variation) } \\
\hline Microcitrus australasica & 30 & 40 & 36 & 31 & 30 \\
\hline \multirow{3}{*}{$\begin{array}{l}\text { Australian finger lime } \\
\text { (1484) }\end{array}$} & 36 & 40 & 40 & 40 & 40 \\
\hline & 40 & 35 & 40 & 40 & 40 \\
\hline & 40 & 40 & 40 & 40 & 35 \\
\hline \multicolumn{6}{|c|}{ Category 4 (tolerant: recovery from infection) } \\
\hline Balsamocitrus daweii & 40 & 30 & 40 & 40 & 40 \\
\hline \multirow{3}{*}{$\begin{array}{l}\text { Uganda powder flask } \\
\text { (3514) }\end{array}$} & 40 & 33 & 40 & 40 & 40 \\
\hline & 28 & 30 & $D^{*}$ & & \\
\hline & 40 & 35 & 40 & 40 & 40 \\
\hline \multicolumn{6}{|c|}{ Category 5 (tolerant: delayed infections) } \\
\hline Poncirus trifoliata & 40 & 40 & 26 & 28 & 30 \\
\hline \multirow[t]{3}{*}{ var. Little-leaf (4007) } & 37 & 40 & 40 & 40 & 34 \\
\hline & 40 & 38 & 30 & 30 & 29 \\
\hline & 40 & 40 & 30 & 28 & 29 \\
\hline \multicolumn{6}{|c|}{ Category 6 (susceptible, retained leaves) } \\
\hline Citrus medica & 24 & 32 & 27 & 29 & 30 \\
\hline \multirow[t]{3}{*}{ 'Diamante citron (3523) } & 27 & 26 & 31 & 29 & 33 \\
\hline & 28 & 26 & 28 & 30 & 30 \\
\hline & 24 & 29 & 29 & 29 & 28 \\
\hline \multicolumn{6}{|c|}{ Category 7 (susceptible, died after four years) } \\
\hline Citrus sinensis & 40 & 28 & 23 & 23 & $\mathrm{D}^{*}$ \\
\hline \multirow{3}{*}{$\begin{array}{l}\text { Pineapple sweet orange } \\
\text { (3858) }\end{array}$} & 40 & 31 & 26 & 26 & \\
\hline & 40 & 25 & 25 & $\mathrm{D}^{* *}$ & \\
\hline & 24 & 26 & $\mathrm{D}^{* *}$ & & \\
\hline \multicolumn{6}{|c|}{ Category 8 (susceptible, died in two years) } \\
\hline Citrus reticulata & 29 & $\mathrm{D}^{* *}$ & & & \\
\hline \multirow[t]{3}{*}{ Unnamed mandarin (3812) } & 35 & 24 & $\mathrm{D}^{* *}$ & & \\
\hline & 40 & 25 & $\mathrm{D}^{* *}$ & & \\
\hline & 31 & 22 & $\mathrm{D} * *$ & & \\
\hline
\end{tabular}

${ }^{a}$ As estimated by real time polymerase chain reaction results shown as cycle threshold $(\mathrm{Ct})$ values. $\mathrm{Ct}$ values show an inverse relationship to the level of the pathogen. A value of 40 indicates no detectable pathogen. Decreasing $\mathrm{Ct}$ values indicate increasing levels of the pathogen. D* indicates death due to environmental factors; $\mathrm{D}^{* *}$ represents death due to HLB. preferred host for ACP (Westbrook et al. 2011), thus, tolerance in this cultivar to HLB may be partially attributed to the psyllid preference factor. The second tolerant accession, a Citrus $\times$ Poncirus hybrid ('X639' trifoliate hybrid) had moderate scores for adult and nymph infestation levels. The susceptible accessions in categories 6, 7, and 8 had adult infestation values ranging from 0.25 ('Koji' mandarin) to 1.11 ('Tien Chieh' mandarin); nymph infestation scores ranged from 0.48 (in Chinese box orange) to 2.09 (in 'Swingle' citrumelo).

\section{Discussion}

In the present study, we have evaluated the disease response of 65 citrus accessions and 33 accessions from 20 citrus-related genera when exposed to natural transmission conditions. As a systematic study using cataloged accessions, we believe that the results obtained will be valuable to validate the HLB host plant lists, to formulate regulations aimed toward disease control, to alleviate HLB epidemics in regions where the disease is not yet widespread, and-most importantly - to identify tolerant and resistant cultivars of Citrus and other related genera. The resistant plants will be useful in the future to understand mode of resistance and to characterize genes associated with resistance. This information may be utilized to generate resistant commercial cultivars, either by conventional breeding or by transgenic approaches. The citrus accessions chosen for the study are presumed to represent the genetic diversity in the genus Citrus from the collection maintained at Riverside, CA (Barkley 2003). The field trial was conducted in the HLB-endemic region of southeastern Florida, where a high psyllid population has established naturally. The type of rootstocks used for citrus cultivation are known to have an effect on HLB disease incidence in the scion cultivars (Albrecht and

Table 4. Huanglongbing disease categories in different genera tested in this study ${ }^{\mathrm{a}}$

\begin{tabular}{|c|c|c|c|}
\hline No. & $\begin{array}{c}\text { Genus (accessions } \\
\text { tested) }\end{array}$ & $\begin{array}{c}\text { Taxonomical } \\
\text { grouping }\end{array}$ & Disease category \\
\hline 1 & Casimiroa (1) & Toddalioideae & 2 \\
\hline 2 & Zanthoxylum (1) & Toddalioideae & 2 \\
\hline 3 & Bergera sp. (1) & $\begin{array}{l}\text { Aur/Clauseneae/ } \\
\text { Clauseninae }\end{array}$ & 2 \\
\hline 4 & Clausena (2) & $\begin{array}{l}\text { Aur/Clauseneae/ } \\
\text { Clauseninae }\end{array}$ & 1 and * \\
\hline 5 & Glycosmis (1) & $\begin{array}{l}\text { Aur/Clauseneae/ } \\
\text { Clauseninae }\end{array}$ & 1 \\
\hline 6 & Murraya (2) & $\begin{array}{l}\text { Aur/Clauseneae/ } \\
\text { Clauseninae }\end{array}$ & 2 and 4 \\
\hline 7 & Aegle (1) & $\begin{array}{l}\text { Aur/Citreae/ } \\
\text { Balsamocitrinae }\end{array}$ & 4 \\
\hline 8 & Aeglopsis (1) & $\begin{array}{l}\text { Aur/Citreae/ } \\
\text { Balsamocitrinae }\end{array}$ & 4 \\
\hline 9 & Afraegle (1) & $\begin{array}{l}\text { Aur/Citreae/ } \\
\text { Balsamocitrinae }\end{array}$ & * \\
\hline 10 & Balsamocitrus (1) & $\begin{array}{l}\text { Aur/Citreae/ } \\
\text { Balsamocitrinae }\end{array}$ & 4 \\
\hline 11 & Atalantia (1) & Aur/Citreae/Citrinae & $*$ \\
\hline 12 & Citropsis (1) & Aur/Citreae/Citrinae & $*$ \\
\hline 13 & Eremocitrus (1) & Aur/Citreae/Citrinae & 2 \\
\hline 14 & Fortunella (1) & Aur/Citreae/Citrinae & $*$ \\
\hline 15 & Microcitrus (4) & Aur/Citreae/Citrinae & $\begin{array}{l}3 \text { and } 4 \text { ( } 2 \text { accessions } \\
\text { each) }\end{array}$ \\
\hline 16 & Naringi (1) & Aur/Citreae/Citrinae & $2 *$ \\
\hline 17 & Poncirus (2) & Aur/Citreae/Citrinae & 3 and 5 \\
\hline 18 & Severinia (5) & Aur/Citreae/Citrinae & 6 and $*$ \\
\hline 19 & XCitroncirus (4) & Aur/Citreae/Citrinae & $\begin{array}{l}5(1 \text { accession }) \\
6(3 \text { accession })\end{array}$ \\
\hline 20 & XMicrocitronella (1) & Aur/Citreae/Citrinae & $3 *$ \\
\hline 21 & Citrus (65) & Aur/Citreae/Citrinae & $\begin{array}{l}3 \text { (1 accession) } \\
4 \text { (1 accession) } \\
6 \text { (32 accessions) } \\
7 \text { (25 accessions) } \\
8 \text { (6 accessions })\end{array}$ \\
\hline
\end{tabular}

${ }^{a}$ Aur $=$ Aurantioideae; asterisks $(*)$ indicate insufficient data. 
Bowman 2012; Shokrollah et al. 2011). Since the test plants in this field trial were grown as seedling trees without any rootstock, we were able to evaluate the cultivars without the confounding effects of rootstocks. Sample collections were conducted during October or November, since the bacterial titer is known to be highest during these periods in Florida (Manjunath et al. 2008; Wang et al. 2006).

In the current field trial, the experimental seedlings were raised from open-pollinated seeds collected from trees growing in a grove with multiple varieties from the subfamily Aurantioideae. Since the seed parent is known, the eight seedlings may represent half-sib families. If the seed parent is monoembryonic, the eight plants tested are likely to be genetically different from each other, each being a unique hybrid with possibly different pollen parents. Since some citrus is self-compatible, it is also possible that seedlings of some accessions result from self-pollination. If the seed parent has a high degree of nucellar embryony, it is likely that the eight seedlings are clones of the seed parent and represent replicate plants. In accessions with nucellar embryony, certain seedlings may be from zygotic embryos, while others may be nucellar clones of the seed parent (Koltunow et al. 1996). Nucellar seedlings do not develop if pollination does not occur, since the endosperm fails, and its extent is cultivardependent (Altaf et al. 2001; Khan and Roose 1988; Webber 1930; Wright 1937). Information on the occurrence of nucellar embryony in many accessions included in this study is not available. The percentage of nucellar embryos will vary depending on the pollen parent, type of pollinator, and environmental factors (Mondal and Saha 2014). Generally, citrons and pummelos do not have nucellar embryony (Kishore et al. 2012). Many mandarins, sour oranges, sweet oranges, lemons, limes, grapefruit, and trifoliates are known to possess nucellar embryony (Nakano et al. 2012; Soost and Roose 1996). Among the citrus relatives included in the study, Indian bael has been shown to have nucellar embryony (Johri and Ahuja 1957). Curry leaf plant has been reported to have polyembryony, which predicts the occurrence of nucellar embryony (Madalageri and Gowda 1987). Table 2 indicates accessions in which the nucellar embryony or monoembryony status is known. Certain accessions are presumed to have monoembryony or nucellar embryony based on group characters. Even when the nucellar embryony status of a cultivar is known, it is difficult to speculate if the eight seedlings of an accession used in our trials arose as nucellar seedlings or if they are zygotic seedlings. In one of the categories we identified, the HLB-tolerant plants appear to show seedling variations (category 3). Out of the six accessions in this category, three are presumed to be monoembryonic (Australian finger lime, 'Sydney hybrid', and Large-leaf Australian wild lime) and one accession is probably polyembryonic ('Simmon's trifoliate'); the status of 'Mountain' citron and 'Faustrimedin' is not known. The polyembryonic 'Simmon's trifoliate' probably has seedlings of different genotypes, since we clearly saw that some seedlings were susceptible while others were tolerant to HLB. In Microcitrus spp., nucellar embryony has not been reported and we assumed that the accessions are monoembryonic. Two types of seedlings (susceptible and tolerant) seem to be present in the progeny from Microcitrus spp. and citrus hybrids with Microcitrus parentage.

Plants can develop innate immunity because of structural, chemical, or protein-based defenses (Doughari 2015). In the present study, only two accessions (from a total of 90) are totally immune to the HLB pathogen (Table 1). The genus Clausena has several species that are commonly grown in China and South East Asia. Classical HLB symptoms have been described in Clausena lansium Skeels growing in the Guangdong province of China (Deng et al. 2007). The infected tree in China was reported to have yellow shoots typical of HLB. However, only very low titers of the pathogen were recorded and ' $\mathrm{Ca}$. L. asiaticus' could only be detected by nested PCR (Deng et al. 2007; Ding et al. 2005). The cataloged accession of Clausena excavata was found to be immune to the Florida ' $\mathrm{Ca}$. L. asiaticus' strain in the current study.

Resistance to disease is invaluable for the successful management of citrus HLB. Six accessions in the study were considered as HLBresistant hosts (category 2). Unlike the immune accessions, the resistant taxa were capable of supporting multiplication of the pathogen transiently to various levels, as shown in this study. Although the pathogen was present for only a short duration, it is possible for a psyllid feeding on such a plant to acquire the pathogen and transmit it to a more receptive host plant, such as Citrus spp. (Lee et al. 2015). Our data clearly showed that the pathogen always disappeared in the resistant types and did not colonize the plant host. From a regulatory point of view, plants categorized as resistant in our study may need to be included in the list of regulated plants, especially in regions where HLB is in the initial stages of the imminent epidemic, to avoid disease spread via alternate plant hosts.

White sapote is not a preferred host for the psyllid (Halbert and Manjunath 2004; Westbrook et al. 2011). Since this plant is grown in California by rare fruit growers and horticulturists, mainly in regions where citrus is cultivated, and it may act as an alternate host for HLB, regulatory precautions are warranted. Greening-associated ' $\mathrm{Ca}$. L. asiaticus' or disease symptoms have never been reported from curry leaf. Hung et al. (2000) extracted DNA from greenhouse-grown curry leaf plants that were exposed to HLB and hybridized to ' $\mathrm{Ca}$. L. asiaticus' nucleic acid. ' $C a$. L. asiaticus' DNA was not detected in these experiments, indicating absence of ' $\mathrm{Ca}$. L. asiaticus' in curry leaf (Hung et al. 2000). Transmission of HLB from citrus to curry leaf has not been successful under greenhouse conditions (Damsteegt et al. 2010; Hung et al. 2000). Conflicting reports exist regarding the susceptibility or resistance of Murraya paniculata to citrus HLB (Hung et al. 2000, 2001; Lopes et al. 2010). In the current field trial, the 'Orange jasmine' trees were very healthy-looking, while 'Hawaiian mock orange' showed some chlorotic branches. In other studies, yellow shoots and insignificant blotchy mottle have been reported from Murraya paniculata (Nguyen 2012). Since curry leaf and 'Orange jasmine' are preferred hosts of ACP, the chances of pathogen spread (liberibacter that may be transiently present in the plant) through psyllids would be potentially high. Large ACP populations have been recorded from urban plantings of $M$. paniculata in Florida (Hall and Rohrig 2015). Transmission experiments showed that the pathogen could be transmitted experimentally from 'Orange jasmine' to citrus (Damsteegt et al. 2010; Tirtawidjaja 1981). The Asian greening-associated pathogens in Taiwan do not multiply either in Murraya paniculata or in Bergera spp. (Hung et al. 2000; Toorawa 1998). Surveys by Walter et al. (2012a and b) of $M$. paniculata plantings in Florida indicated that the incidence of the HLBassociated pathogen was extremely low, both in the plants and in ACP infesting the plants.

We used the ' $\mathrm{Ca}$. L. asiaticus' $\mathrm{Ct}$ values to determine and define the disease response categories (Table 1). Some of the samples with high $\mathrm{Ct}$ value may actually be negative for ' $\mathrm{Ca}$. L. asiaticus'. Since one molecule of target DNA under ideal conditions of real time PCR should give a Ct value of about 37 (G. McCollum, personal communication), DNA deposited by psyllids while feeding or the presence of dead bacteria may result in a positive $\mathrm{Ct}$ value $(>37$ and $<40$ ), even if there is no replication. It has been demonstrated that acquisition of ' $\mathrm{Ca}$. L. asiaticus' is more efficient when a psyllid nymph feeds on an infected plant than when an adult psyllid feeds (Inoue et al. 2009; Pelz-Stelinski et al. 2010). Based on this criterion, Zanthoxylum spp. may not be an alternate host for HLB, since the psyllid does not colonize on this accession.

Australian desert lime (Eremocitrus glauca hybrid) was highly resistant to ' $\mathrm{Ca}$. L. asiaticus' in our trials, however, there are no published reports on the HLB host status for Eremocitrus spp. It is likely that since ' $\mathrm{Ca}$. L. asiaticus' has never encountered this host in its natural habitat in Australia, it may lack adaptation to infect Australian desert lime. Ongoing studies with both Eremocitrus glauca (Citrus Research Center number CRC 4107) and some of the available natural hybrids of E. glauca (with citrus) have confirmed both the existence of a high level of resistance in E. glauca as well as the inheritance of resistance in hybrid accessions (data not shown).

In category 3 , seedling variations in plants within an accession resulted in two different types of responses to HLB challenge - susceptibility to the disease in some seedling trees and a high degree of resistance in some other seedlings. This may arise due to the genetic variability in the open-pollinated seeds or due to segregation of traits in hybrid 
accessions. Some seedlings of 'Sydney hybrid' showed a high titer of ' $C a$. L. asiaticus' and severe stunting, while others showed normal growth and no detectable ' $\mathrm{Ca}$. L. asiaticus'. Three species of genus Microcitrus and two hybrids having Microcitrus parentage were evaluated in the study. Genus Microcitrus is presumed to be monoembryonic and the seedlings are likely to be genetically different from each other. In spite of having a high ' $\mathrm{Ca}$. L. asiaticus' titer (Ct values of 24 to 30 recorded in many), plants with Microcitrus parentage displayed good field tolerance. Typical HLB symptoms are not seen on Australian citrus types. The die-back of the shoots observed in Microcitrus spp. may be associated with HLB infection. Psyllids can feed and colonize well on all of the Microcitrus accessions included in the study. It may be prudent to include them on the exclusion list for HLB quarantine purposes.

'Simmon's trifoliate' showed resistance to HLB in five out of eight seedlings. Since this accession may have polyembryony, it may be interesting to analyze resistant and susceptible seedlings. Trifoliates have been shown to have more resistance to HLB than other citrus groups (Albrecht and Bowman 2011, 2012; Boava et al. 2015; Folimonova et al. 2009). Six accessions with trifoliate parentage were included in the current study. Among the Poncirus trifoliata accessions, 'Simmon's trifoliate' was HLB tolerant (category 3); 'Little-leaf' trifoliate was more susceptible (category 5), in spite of the psyllid abundance and colonization scores being low (Aubert 1987; 1990; Richardson and Hall 2013; Westbrook et al. 2011). In addition to effects on psyllid feeding behavior, antibacterial compounds associated with the Poncirus trifoliata parentage may be responsible for disease tolerance (Albrecht and Bowman 2011). The two Poncirus accessions studied are resistant to ACP (Westbrook et al. 2011) and tolerant to HLB. Interestingly, HLB tolerance recorded in Poncirus trifoliata accessions was not observed in four hybrids of Citrus and Poncirus tested in this study, 'Rusk' citrange, 'S-281' citrangelo, 'Swingle' citrumelo $(C$. paradisi Duncan $\times P$. trifoliata), and 'X639' (Citrus reshni Cleopatra mandarin $\times$ Poncirus trifoliata).

Even though, we have categorized many accessions as resistant or highly tolerant and placed them in categories 2 and 3, many of them support transient replication of ' $\mathrm{Ca}$. L. asiaticus' to varying levels. While these accessions may have utility in breeding for disease resistance, they should be considered as potential alternate hosts for regulatory purposes, especially in regions like California, where the disease has not yet established. In the United States, prior to 2008, genus Murraya was not regulated and there was free movement of ornamental Murraya spp. throughout the state of Florida (Halbert et al. 2012). Based on a survey conducted during 2005 and 2006, we reported ' $\mathrm{Ca}$. L. asiaticus'-positive nymphs (with Ct values as low as 22) collected from ornamental Murraya plants marked for sale in big box stores located in Nassau county in the northern part of Florida, where commercial citrus was not grown (Manjunath et al. 2008). Positive psyllid nymphs indicate presence of the pathogen in the plant on which the nymphs are feeding. The origin of these Murraya plants was traced to Miami Dade County, where HLB was reported at that time. Tens of thousands of potted Murraya plants were exported out of the Miami Dade area during this period (Halbert et al. 2012). It is likely that unregulated movement of Murraya spp. during the early stages of the epidemic might have contributed toward widespread dispersal of the disease in Florida (Manjunath et al. 2008). Similar observations were made by Lopes et al. (2010) regarding the spread of HLB through the movement of Murraya plants in Brazil. Transmission of HLB from Murraya plants to Citrus plants has been demonstrated (Damsteegt et al. 2010; Zhou et al. 2007). The disease response of different Murraya cultivars to various liberibacter isolates are known to vary (Lopes et al. 2010; Tirtawidjaja 1981). In this study, the two accessions of Murraya spp. showed resistant and tolerant responses to the pathogen in the field. The low ' $\mathrm{Ca}$. L. asiaticus' titers we observed in Murraya spp. agrees with similar results reported by other researchers (Damsteegt et al. 2010; Walter et al. 2012a and b).

The Australian limes (genera Eremocitrus and Microcitrus) are close relatives of citrus but are morphologically distinct and are presumed to have diverged from genus Citrus about 3.5 million years ago (Schwartz et al. 2015). According to some taxonomists, they are grouped under the genus Citrus (Mabberley 1997). In the subtribe Citrinae, five of the six genera (Citrus, Poncirus, Fortunella, Microcitrus, and Eremocitrus) are known to be sexually compatible with each other (Iwamasa et al. 1988). The high degree of resistance found during this study in genus Poncirus and the two Australian genera Eremocitrus and Microcitrus is probably of great value, since they can be crossed with citrus. It may be possible to develop HLBresistant hybrid rootstocks and scions using these as parents in breeding trials. Hybrid populations may also serve as excellent resources for marker analysis and biochemical and molecular characterization of resistance mechanisms. Other sexually noncompatible members of Aurantioideae may have different mechanisms of resistance and may be utilized for HLB mitigation. Certain graft-compatible accessions may be able to provide good resistance if used as approach grafts. Planting citrus with two rootstocks, while expensive, may be worthwhile as an interim approach, if resistance to HLB can be obtained.

\section{Acknowledgments}

We gratefully acknowledge the Citrus Research Board (project number 5300-123) for financial support. Excellent technical support was provided by E. Rodriguez and H. Park from the University of California, Riverside and C. Latza from the U.S. Horticultural Laboratory, Fort Pierce. The field planting was established and maintained by S. Ciliento, D. Helseth, S. Mayo, S. Reif, and G. Swearingen from the U.S. Horticultural Laboratory, Fort Pierce. This is Entomology Contribution No. 1299, Florida Department of Agriculture and Consumer Services, Division of Plant Industry, Bureau of Entomology, Nematology, and Plant Pathology.

\section{Literature Cited}

Ahmad, K., Sijam, K., Hashim, H., Kadir, J., and Rastan, S. O. S. 2008. Occurrence and spread of Candidatus Liberibacter asiaticus, the causal agent of huanglongbing disease of citrus in Malaysia. Res. J. Agric. Biol. Sci. 4:103-111.

Albrecht, U., and Bowman, K. D. 2011. Tolerance of the trifoliate citrus hybrid US-897 (Citrus reticulata Blanco $\times$ Poncirus trifoliata L. raf.) to huanglongbing. HortScience 46:16-22.

Albrecht, U., and Bowman, K. D. 2012. Tolerance of trifoliate rootstock hybrids to Candidatus Liberibacter asiaticus. Sci. Hortic. (Amsterdam) 147:71-80.

Altaf, N., Murwat, E. K., Bhatti, I. A., and Iqbal, M. M. 2001. Nucellar regeneration and polyembryony of citrus cultivars. Pak. J. Bot. 33:211-215.

Aubert, B. 1987. Trioza erytreae del Guercio and Diaphorina citri Kuwayama (Homoptera: Psylloidea), the two vectors of citrus greening disease: Biological aspects and possible control strategies. Fruits 42:149-162.

Aubert, B. 1990. Integrated activities for the control of huanglongbing-greening and its vector Diaphorina citri Kuwayama in Asia. Page 133-144 in: Rehabilitation of Citrus Industry in the Asia Pacific region. B. Aubert, S. Tontyaporn, and D. Buangsuwon, eds. Proceedings of Asia Pacific International conference on Citriculture, Chiang Mai, Thailand, February 4-10 1990. United Nations Development Programme-Food and Agriculture Organization, Rome.

Barkley, N. A. 2003. Genetic diversity in a citrus germplasm collection characterized with simple sequence repeat markers. Ph.D. thesis. University of California, Riverside, CA.

Beattie, G. A. C., Holford, P., Haigh, A. M., Nguyen, H. C., Mabberley, D. J., Weston, P. H., Broadbent, P., and Spooner-Hart, R. N. 2012. Huanglongbing: Research and insights from collaborative research in Southeast Asia. Presentation. International symposium on Epidemiology and Disease Management of Citrus Huanglongbing Disease for Sustainable Citrus Production in the ASPAC Region. Nov 5-12, 2012. National Taiwan University, Taipei, Taiwan.

Boava, L. P., Sagawa, C. H. D., Cristofani-Yaly, M., and Machado, M. A. 2015 Incidence of 'Candidatus Liberibacter asiaticus' infected plants among citrandarins as rootstock and scion under field conditions. Phytopathology 105:518-524

Bové, J. M. 2006. Huanglongbing: A destructive, newly emerging, century-old disease of citrus. J. Plant Pathol. 88:7-37.

Chen, Q. 1943. A report of a study on yellow shoot of citrus in Chaoshan. N. Agric Q. Bull. 3:142-175.

Coletta-Filho, H. D., Targon, M. L. P. N., Takita, M. A., De Negri, J. D., Pompeu, J., Jr., Machado, M. A., do Amaral, A. M., and Muller, G. W. 2004. First report of the causal agent of huanglongbing ("Candidatus Liberibacter asiaticus") in Brazil. Plant Dis. 88:1382.

da Graça, J. V., and Korsten, L. 2004. Citrus huanglongbing: Review, present status and future strategies. Pages 229-245 in: Diseases of Fruits and Vegetables. S. A. M. H. Naqvi, ed. Kluwer Academic Publishers, Dordrecht, The Netherlands.

Damsteegt, V. D., Postnikova, E. N., Stone, A. L., Kuhlmann, M., Wilson, C., Sechler, A., Schaad, N. W., Brlansky, R. H., and Schneider, W. L. 2010 Murraya paniculata and related species as potential hosts and inoculum 
reservoirs of 'Candidatus Liberibacter asiaticus', causal agent of huanglongbing. Plant Dis. 94:528-533.

Deng, X., Zhou, G., Li, H., Chen, J., and Civerolo, E. L. 2007. Detection of Candidatus Liberibacter asiaticus from wampee (Clausena lansium Skeels) by nested PCR. Plant Health Prog. Published online. doi:10.1094/PHP-20070419-01-BR

Ding, F., Wang, G., Yi, G., Zhong, Y., Zeng, J., and Zhou, B. 2005. Infection of Wampee and lemon by the citrus huanglongbing pathogen (Candidatus Liberibacter asiaticus) in China. J. Plant Pathol. 87:207-212.

Doughari, J. H. 2015. An overview of plant immunity. J. Plant Pathol. Microbiol. $6: 322$.

Folimonova, S. Y., Robertson, C. J., Garnsey, S. M., Gowda, S., and Dawson, W. O. 2009. Examination of the responses of different genotypes of citrus to huanglongbing (citrus greening) under different conditions. Phytopathology 99:1346-1354.

Ford-Lloyd, B. V., Schmidt, M., Armstrong, S. J., Barazani, O., Engels, J., Hadas, R., Hammer, K., Kell, S. P., Kang, D., Khoshbakht, K., Li, Y., Long, C., Lu, B.-R., Ma, K., Nguyen, V. T., Qiu, L., Ge, S., Wei, W., Zhang, Z., and Maxted, N. 2011. Crop wild relatives-Undervalued, underutilized and under threat? Bioscience 61:559-565.

Gottwald, T. R. 2010. Current epidemiological understanding of citrus huanglongbing. Annu. Rev. Phytopathol. 48:119-139.

Guajardo, V., Hinrichsen, P., and Muñoz, C. 2015. Breeding rootstocks for Prunus species: Advances in genetic and genomics of peach and cherry as a model. Chil. J. Agric. Res. 75:17-27.

Halbert, S. E. 2005. The discovery of huanglongbing in Florida. In: Proceedings of the 2nd International Citrus Canker and Huanglongbing Research Workshop, Nov. 7-11, 2005. T. R. Gottwald, W. N. Dixon, J. H. Graham, and P. Berger, eds. Florida Citrus Mutual, Orlando, FL. Paper H-3.

Halbert, S. E., and Manjunath, K. L. 2004. Asian citrus psyllids (Sternorrhyncha: Psyllidae) and greening disease of citrus: A literature review and assessment of risk in Florida. Fla. Entomol. 87:330-353.

Halbert, S. E., Manjunath, K., Ramadugu, C., and Lee, R. F. 2012. Incidence of huanglongbing-associated 'Candidatus Liberibacter asiaticus' in Diaphorina citri (Hemiptera: Psyllidae) collected from plants for sale in Florida. Fla. Entomol. 95:617-624.

Hall, D. G., and Rohrig, E. 2015. Bionomics of Asian citrus psyllid (Hemiptera: Liviidae) associated with orange jasmine hedges in southeast central Florida, with special reference to biological control by Tamarixia radiata. J. Econ. Entomol. 108:1198-1207.

Hall, D. G., George, J., and Lapointe, S. L. 2015. Further investigations on colonization of Poncirus trifoliata by the Asian citrus psyllid. Crop Prot. 72: 112-118.

Holland, J. B. 2004. Breeding: Incorporation of exotic germplasm. Encyclopedia of Plant and Crop Science. Marcel Dekker, Inc., New York.

Hu, H., Roy, A., and Brlansky, R. H. 2014. Live population dynamics of 'Candidatus Liberibacter asiaticus', the bacterial agent associated with citrus huanglongbing, in citrus and non-citrus hosts. Plant Dis. 98: 876-884.

Hung, T. H., Wu, M. L., and Su, H. J. 2000. Identification of alternate hosts of the fastidious bacterium causing citrus greening disease. J. Phytopathol. 148: 321-326.

Hung, T. H., Wu, M. L., and Su, H. J. 2001. Identification of the Chinese box orange (Severinia buxifolia) as an alternative host of the bacterium causing citrus Huanglongbing. Eur. J. Plant Pathol. 107:183-189.

Husain, M.A. and Nath, D. 1927. The citrus psylla (Diaphorina citri, Kuw.) [Psyllidae: Homoptera]. Page 5-27 in: Memoirs of the Department of Agriculture in India, Entomological Series 10. Indian Agricultural Research Institute, Calcutta.

Inch, S., Stover, E., Driggers, R., and Lee, R. F. 2014. Freeze response of Citrus and Citrus-related genotypes in a Florida field planting. HortScience 49: 1010-1016.

Inoue, H., Ohnishi, J., Ito, T., Tomimura, K., Miyata, S., Iwanami, T., and Ashihara, W. 2009. Enhanced proliferation and efficient transmission of Candidatus Liberibacter asiaticus by adult Diaphorina citri after acquisition feeding in the nymphal stage. Ann. Appl. Biol. 155:29-36.

Iwamasa, M., Nito, N., and Ling, J.-T. 1988. Intra- and intergeneric hybridization in the orange subfamily, Aurantioideae. Pages 123-130 in: Proceedings of the Sixth International Citrus Congress. R. Goren and K. Mendel, eds. Margraf Publishers, Weikersheim, FRG. March 6-11, 1988.

Johri, B. M., and Ahuja, M. R. 1957. A contribution to the floral morphology and embryology of Aegle marmelos Correa. Phytomorphology 7:10-24.

Khan, I. A., and Roose, M. L. 1988. Frequency and characteristics of nucellar and zygotic seedlings in three cultivars of trifoliate orange. J. Am. Soc. Hortic. Sci. 113:105-110.

Kishore, K., Monika, N., Rinchen, D., Lepcha, B., and Pandey, B. 2012. Polyembryony and seedling emergence traits in apomictic citrus. Sci. Hortic. (Amsterdam) 138:101-107.

Koizumi, M., Prommintara, M., Linwattana, G., and Kaisuwan, T. 1993. Field evaluation of citrus cultivars for greening resistance in Thailand. Pages 274-279 in: Proceedings of the $12^{\text {th }}$ Conference of the International Organization of Citrus Virologists (IOCV). P. Moreno, J.V. da Graça, and L.W. Timmer, eds. University of California, Riverside, CA.
Koizumi, M., Prommintara, M., and Ohtsu, Y. 1996. Wood apple, Limonia acidissima: A new host for the huanglongbing (greening) vector, Diaphorina citri. Pages 271-275 in: Proceedings of the $13^{\text {th }}$ Conference of the International Organization of Citrus Virologists (IOCV). J.V. da Graça, P. Moreno, and R. K. Yokomi, eds. University of California, Riverside, CA

Koltunow, A. M., Hidaka, T., and Robinson, S. P. 1996. Polyembryony in Citrus. Accumulation of seed storage proteins in seeds and in embryos cultured in vitro. Plant Physiol. 110:599-609.

Lee, J. A., Halbert, S. E., Dawson, W. O., Robertson, C. J., Keesling, J. E., and Singer, B. H. 2015. Asymptomatic spread of huanglongbing and implications for disease control. Proc. Natl. Acad. Sci. U.S.A. 112:7605-7610.

Li, W., Hartung, J. S., and Levy, L. 2006. Quantitative real-time PCR for detection and identification of 'Candidatus Liberibacter species' associated with citrus huanglongbing. J. Microbiol. Methods 66:104-115.

Lopes, S. A., Frare, G. F., Camargo, L. E. A., Wulff, N. A., Teixeira, D. C., Bassanezi, R. B., Beattie, G. A. C., and Ayres, A. J. 2010. Liberibacters associated with orange jasmine in Brazil: Incidence in urban areas and relatedness to citrus liberibacters. Plant Pathol. 59:1044-1053.

Mabberley, D. J. 1997. A classification for edible Citrus (Rutaceae). Telopea (Syd.) 7:167-172

Madalageri, B. B., and Gowda, P. H. R. 1987. First report on polyembryony in curry leaf (Murraya koenigii). Curr. Sci. 56:1286-1287.

Manjunath, K. L., Halbert, S. E., Ramadugu, C., Webb, S., and Lee, R. F. 2008 Detection of 'Candidatus Liberibacter asiaticus' in Diaphorina citri and its importance in the management of citrus huanglongbing in Florida. Phytopathology 98:387-396

Mondal, B., and Saha, R. 2014. Identification of zygotic and nucellar seedlings of Citrus reticulata and Citrus aurantifolia using RAPD. Int. J. Adv. Biotechnol. Res. 5:25-30

Nakano, M., Shimada, T., Endo, T., Fujii, H., Nesumi, H., Kita, M., Ebina, M., Shimizu, T., and Omura, M. 2012. Characterization of genomic sequence showing strong association with polyembryony among diverse Citrus species and cultivars, and its synteny with Vitis and Populus. Plant Sci. 183:131-142

Nguyen, H. C. 2012. Circumscription of Murraya and Merrillia (Sapindales: Rutaceae: aurantioideae) and susceptibility of species and forms to huanglongbing. Ph.D. thesis, University of Western Sydney, Australia.

Pelz-Stelinski, K. S., Brlansky, R. H., Ebert, T. A., and Rogers, M. E. 2010. Transmission parameters for Candidatus Liberibacter asiaticus by Asian citrus psyllid (Hemiptera: Psyllidae). J. Econ. Entomol. 103:1531-1541.

Razi, M. F., Keremane, M. L., Ramadugu, C., Roose, M., Khan, I. A., and Lee, R. F. 2014. Detection of citrus huanglongbing-associated 'Candidatus Liberibacter asiaticus' in citrus and Diaphorina citri in Pakistan, seasona variability, and implications for disease management. Phytopathology 104: 257-268.

Richardson, M. L., and Hall, D. G. 2013. Resistance of Poncirus and Citrus $\times$ Poncirus germplasm to the Asian citrus psyllid. Crop Sci. 53:183-188.

Richardson, M. L., Westbrook, C. J., Hall, D. G., Stover, E., Duan, Y. P., and Lee, R. F. 2011. Abundance of citrus leafminer larvae on Citrus and Citrus-related germplasm. HortScience 46:1260-1264.

Schwartz, T., Nylinder, S., Ramadugu, C., Antonelli, A., and Pfeil, B. E. 2015 The origin of oranges: A multi-locus phylogeny of Rutaceae subfamily Aurantioideae. Syst. Bot. 40:1053-1062.

Sétamou, M., da Graça, J. V. and Sandoval, II, J. L. 2016. Suitability of native North American Rutaceae to serve as host plants for the Asian citrus psyllid (Hemiptera: Liviidae). J. Appl. Entomol. doi: 10.1111/jen.12300

Shokrollah, H., Abdullah, T. L., Sijam, K., and Abdullah, S. N. A. 2011. Potential use of selected citrus rootstocks and interstocks against HLB disease in Malaysia. Crop Prot. 30:521-525.

Shokrollah, H., Abdullah, T. L., Sijam, K., Abdullah, S. N. A., and Abdullah, N. A. P. 2009. Differential reaction of citrus species in Malaysia to huanglongbing (HLB) disease using grafting method. Am. J. Agric. Biol. Sci. 4:32-38.

Soost, R. K., and Roose, M. L. 1996. Citrus. Pages 257-323 in: Fruit Breeding, Vol. I: Tree and Tropical Fruits. J. Janick and J. N. Moore, eds. John Wiley \& Sons, Inc., New York.

Stover, E., and McCollum, G. 2011. Incidence and severity of huanglongbing and Candidatus Liberibacter asiaticus titer among field-infected citrus cultivars. HortScience 46:1344-1348.

Stover, E., McCollum, G., Ramos, J., and Shatters, R. G. 2014. Growth, health and Liberibacter asiaticus titer in diverse citrus scions on mandarin versus trifoliate hybrid rootstocks in a field planting with severe huanglongbing. Proc. Fla. State Hortic. Soc. 127:53-59.

Tirtawidjaja, S. 1981. Insect, dodder and seed transmissions of citrus vein phloem degeneration (CVPD). In: Proceedings of the Fourth International Society of Citriculture Congress. Tokyo, Japan. International Society of Citriculture, pp 469-471.

Toorawa, P. 1998. La maladie du huanglongbing (greening) des agrumes a L'île Maurice. Detection de "Candidatus Liberobacter asiaticum" dans les agrumes et les insects vecteurs. Ph.D. thesis. L'University de Bordeaux, France.

Tsai, J. H., Wang, J. J., and Liu, Y. H. 2002. Seasonal abundance of the Asian citrus psyllid, Diaphorina citri (Homoptera: Psyllidae) in Southern Florida. Fla. Entomol. 85:446-451. 
USDA National Agricultural Statistics Service. 2015. October forecast: Maturity test results and fruit size. United States Department of Agriculture, Washington, D.C. Published online. http://www.nass.usda.gov/Statistics_by_State/Florida/Publications/ Citrus/cit/2015-16/cit1015.pdf.

Vale, F. X. R. D., Parlevliet, J. E., and Zambolim, L. 2001. Concepts in plant disease resistance. Fitopatol. Bras. 26:577-589.

Walter, A. J., Duan, Y. P., and Hall, D. G. 2012a. Titers of ' $C a$. Liberibacter asiaticus' in Murraya paniculata and Murraya-reared Diaphorina citri are much lower than in Citrus and Citrus-reared psyllids. HortScience 47: 1449-1452.

Walter, A. J., Hall, D. G., and Duan, Y. P. 2012b. Low Incidence of Candidatus Liberibacter asiaticus in Diaphorina citri and its host plant Murraya paniculata. Plant Dis. 96:827-832.
Wang, Z., Yin, Y., Hu, H. H., Yuan, Q., Peng, G., and Xia, Y. 2006. Development and application of molecular-based diagnosis for 'Candidatus Liberibacter asiaticus', the causal pathogen of citrus huanglongbing. Plant Pathol. 55:630-638.

Webber, H. J. 1930. Influence of pollination on set of fruit in citrus. Calif Citrograph 15:322-323.

Westbrook, C. J., Hall, D. G., Stover, E., Duan, Y. P., and Lee, R. F. 2011 Colonization of Citrus and Citrus-related germplasm by Diaphorina citri (Hemiptera: Psyllidae). HortScience 46:997-1005.

Wright, N. 1937. Pollination and the seediness of Marsh grapefruit. Proc. Agric. Soc. Trinidad Tobago 37:51-60.

Zhou, L. J., Gabriel, D. W., Duan, Y. P., Halbert, S. E., and Dixon, W. N. 2007. First report of dodder transmission of huanglongbing from naturally infected Murraya paniculata to citrus. Plant Dis. 91:227. 